\title{
PROFIL ISOPÉRIMÉTRIQUE, MÉTRIQUES PÉRIODIQUES ET FORMES D'ÉQUILIBRE DES CRISTAUX
}

\author{
PierRe PANSU ${ }^{1}$
}

\begin{abstract}
An asymptotic expansion of the isoperimetric profile of a periodic Riemannian metric on $\mathbb{R}^{n}$ is given. This has an application to the equilibrium shapes of crystals.

Résumé. On donne un développement asymptotique du profil isopérimétrique de $\mathbb{R}^{n}$ muni d'une métrique riemannienne périodique, et des conséquences pour le problème de la forme d'équilibre des cristaux.
\end{abstract}

AMS Subject Classification. 53C20, 35B27, 52A38, 49Q20.

Reçu le 4 janvier 1999. Révisé le 14 octobre 1999.

\section{INTRODUCTION}

\subsection{Position du problème}

Définition 1. Soit $V$ une variété riemannienne. Pour $\tau>0$, on note $I(\tau)$ la borne inférieure des volumes des bords des sous-variétés compactes de codimension 0 de $V$ de volume $\tau$. La fonction $I$ s'appelle le profil isopérimétrique de $V$.

Soit $T$ un tore riemannien de dimension $n$. On s'intéresse au comportement asymptotique du profil isopérimétrique de son revêtement universel, un espace vectoriel muni d'une métrique périodique. Par comparaison avec une métrique euclidienne, on sait qu'il existe des constantes $c>0$ et $C$ telles que

$$
c \tau^{n-1 / n} \leq I(\tau) \leq C \tau^{n-1 / n}
$$

Il s'agit de préciser cette estimation grossière.

\subsection{Résultats}

Le problème isopérimétrique s'avère relié à la notion de volume asymptotique introduite dans [17], chapitre 4 et développée dans $[2,5,26]$.

Keywords and phrases: Inégalité isopérimétrique, norme stable, homogénéisation, forme de Wulff.

${ }^{1}$ Laboratoire de Mathématique d'Orsay, UMR 8628 du CNRS, bâtiment 425, Université Paris-Sud, 91405 Orsay, France; e-mail:

Pierre.Pansu@math.u-psud.f http://www.math.u-psud.fr/ pansu 
Définition 2. (Version continue). Soit $T$ un tore riemannien de dimension $n, \tilde{T}$ son revêtement universel. On fixe un point $x$ de $\tilde{T}$ et on considère la boule de rayon $r, B(x, r)$. La limite

$$
V A(\tilde{T})=\lim _{r \rightarrow+\infty} r^{-n} \operatorname{Vol} B(x, r)
$$

existe. Elle ne dépend pas de $x$ et s'appelle le volume asymptotique de $\tilde{T}$.

(Version discrète). Soit $E$ un groupe abélien libre de rang $n$ et $N$ une norme sur $E$. La limite

$$
V A(E, N)=\lim _{r \rightarrow+\infty} r^{-n} \#\{e \in E ; N(e) \leq r\}
$$

existe. Elle s'appelle le volume asymptotique de $(E, N)$.

Exemple. Soit $V$ une variété riemannienne de dimension $n$. Pour chaque $k \leq n$, on dispose sur le groupe $E^{k}=H^{k}(V, \mathbb{Z}) /$ tor sion des normes $|\cdot|_{p}$ quotient des normes $L^{p}$ sur les $k$-formes différentielles fermées sur $V$ à périodes entières.

Remarque. La norme $|\cdot|_{\infty}$ est liée à la fameuse norme stable sur l'homologie, voir $[8,15,22]$. En effet, le théorème des coefficients universel établit que $E^{k}=H o m\left(H_{k}(V, \mathbb{Z}), \mathbb{Z}\right)$. Alors la norme stable sur $H_{k}(V, \mathbb{Z})$ est duale de $|\cdot|_{\infty}$.

Théorème 1. Soit $T$ un tore riemannien de dimension $n$. On note I le profil isopérimétrique du revêtement universel de T. La limite

$$
c_{\infty}=\lim _{\tau \rightarrow+\infty} I(\tau) / \tau^{n-1 / n}
$$

existe, elle vaut

$$
c_{\infty}=n V A\left(H^{n-1}(T, \mathbb{Z}),|\cdot|_{\infty}\right)^{1 / n} \operatorname{Vol}(T)^{1-n / n} .
$$

Lorsqu'on fait varier la métrique riemannienne sur $T$, la constante isopérimétrique $c_{\infty}$ varie continument. On peut aisément la rendre arbitrairement petite, en augmentant le volume de $T$ sans changer sensiblement la norme stable sur $H_{n-1}$. En revanche, il est plus difficile de rendre $c_{\infty}$ plus grande. En effet, elle est majorée par un invariant conforme.

Théorème 2. Pour toute métrique riemannienne sur le tore de dimension $n$, on a

$$
c_{\infty} \leq n\left(V A\left(H^{n-1}(T, \mathbb{Z}),|\cdot|_{n / n-1}\right)\right)^{1 / n} \leq n V A(\tilde{T})^{1 / n} .
$$

En particulier, si $n=2$, on a

$$
c_{\infty} \leq \sqrt{4 \pi}
$$

En dimension $n>2$, la borne donnée dans le théorème 2 reste optimale pour les métriques plates. Par conséquent, celles-ci maximisent la fonctionnelle $c_{\infty}$ dans leur classe conforme. Cette observation a été faite indépendamment par Burago et Ivanov [6], ainsi que la suivante.

Proposition 3. Si $n \geq 3$, la constante isopérimétrique $c_{\infty}$ prend des valeurs arbitrairement grandes.

Enfin, nous donnons une seconde estimation dont le cas d'égalité est plus facile à traiter.

Théorème 3. Pour toute métrique riemannienne sur le tore de dimension $n$, on a

$$
c_{\infty} \leq c_{\text {eucl }}\left(\frac{V A\left(H^{n-1}(T, \mathbb{Z}),|\cdot|_{\infty}\right)}{V A\left(H^{1}(T, \mathbb{Z}),|\cdot|_{\infty}\right)^{n-1}}\right)^{1 / n}
$$

où $c_{\text {eucl }}$ est la constante isopérimétrique euclidienne. L'égalité a lieu si et seulement si la métrique est plate. En particulier, un 2-tore riemannien satisfait $c_{\infty}=2 \sqrt{\pi}$ si et seulement si il est plat. 


\subsection{Un problème de cristallographie}

Au siècle dernier, Gauss [16] a proposé de modéliser une goutte (dans le vide sans gravité) d'un liquide incompressible par un domaine à bord lisse de l'espace euclidien, de volume fixé. À l'équilibre, la goutte doit minimiser une énergie de surface proportionnelle à l'aire du bord, c'est pourquoi elle est ronde.

Curie [9] a suggéré de généraliser ce point de vue aux cristaux. Un cristal est modélisé par un polyèdre de l'espace euclidien, dont les faces $f_{i}$ peuvent posséder des constantes capillaires (aujourd'hui, on dirait plutôt tensions superficielles) $C_{i}$ distinctes. L'énergie de surface n'est plus proportionnelle à l'aire du bord, mais à la somme $\sum_{i} C_{i} \operatorname{Aire}\left(f_{i}\right)$. Wulff [34] a montré qu'il existe au plus un polyèdre dont les faces ont des directions fixées et qui minimise l'énergie de surface à volume fixé, c'est la forme de Wulff. Dès que l'on connait les constantes capillaires, on connait donc la forme d'équilibre du cristal.

Plus récemment, la question a été posée d'établir ce résultat à partir de modèles microscopiques. Il y a une littérature abondante sur ce sujet, depuis les travaux de Minlos et Sinai [23] sur le modèle d'Ising, voir par exemple $[1,10-12]$. Ces modèles thermodynamiques incorporent un paramètre supplémentaire, la température.

Voici un modèle naïf de cristal à température nulle. On considère dans l'espace euclidien $\mathbb{R}^{3}$ un réseau de translations $G$ (le "groupe de Bravais") et un sous-ensemble discret $A$ (l'ensemble des atomes ou molécules du cristal infini idéal) invariant par $G$. Pour chaque paire $a_{1}, a_{2}$ de points de $A$, on se donne, de façon invariante par $G$, une énergie d'intéraction $e\left(a_{1}, a_{2}\right)$. Un cristal réel est un sous-ensemble fini $B$ de $A$. On lui associe une énergie de surface, c'est la somme des $e\left(a_{1}, a_{2}\right)$ pour toutes les paires $a_{1}, a_{2}$ telles que $a_{1} \in B$ et $a_{2} \notin B$. En d'autres termes, c'est l'énergie qu'il faut employer à couper toutes les liaisons entre atomes pour découper le morceau $B$.

Définition 4. On appelle norme cristalline la norme sur $\mathbb{R}^{3}$ obtenue par la construction suivante. Soit $\ell$ une forme linéaire sur $\mathbb{R}^{3}$. Un cocycle représentant $\ell$ est une fonction $f$ sur $A$ telle que pour $g \in G$ et $a \in A$, $f(a+g)=f(a)+\ell(g)$. La norme $\ell^{1}$ d'un cocycle $f$ est la somme

$$
\sum_{\left(a_{1}, a_{2}\right) \in A \times A / G} e\left(a_{1}, a_{2}\right)\left|f\left(a_{2}\right)-f\left(a_{1}\right)\right| .
$$

En minimisant la norme $\ell^{1}$ des cocyles représentant une forme linéaire, on obtient une semi-norme $|\cdot|_{\text {cris }}^{*}$ sur le dual de $\mathbb{R}^{3}$. Par définition, la norme cristalline $|\cdot|_{\text {cris }}$ est la norme duale de celle-ci.

Remarque. On montrera (Prop. 42) que la boule unité de la norme cristalline est un polyèdre, éventuellement contenu dans un sous-espace. Si on suppose de plus que les énergies des liaisons sont des nombres rationnels, ce polyèdre est rationnel (les équations des facettes dans une base du groupe $G$ sont à coefficients rationnels). Il y a un algorithme efficace de calcul des facettes.

Définition 5. On définit une distance qui permet de comparer parties finies et parties convexes de l'espace euclidien. Soient $X, X^{\prime}$ deux parties compactes de l'espace euclidien $\mathbb{R}^{n}$. On note $\operatorname{dist}_{H}\left(X, X^{\prime}\right)$ leur distance de Hausdorff, i.e. la borne inférieure des $\epsilon>0$ tels que $X$ soit contenu dans le $\epsilon$-voisinage de $X^{\prime}$ et $X^{\prime}$ dans le $\epsilon$-voisinage de $X$. Soient $\mu$ et $\mu^{\prime}$ deux mesures sur $\mathbb{R}^{n}$. On note $\delta\left(\mu, \mu^{\prime}\right)$ la borne inférieure des nombres

$$
\operatorname{dist}_{H}\left(Y, Y^{\prime}\right)+\mu\left(Y_{c}\right)+\mu^{\prime}\left(Y_{c}^{\prime}\right)
$$

où $Y$ et $Y^{\prime}$ sont des sous-ensembles compacts quelconques de $\mathbb{R}^{n}$ de complémentaires $Y_{c}$ et $Y_{c}^{\prime}$.

Lorsque $B \subset \mathbb{R}^{n}$ est un ensemble fini, on note $\mu_{B}$ la mesure de probabilité équirépartie sur $B$. Lorsque $D \subset \mathbb{R}^{n}$ est un convexe plein d'un sous-espace affine de $\mathbb{R}^{n}$, on note $\mu_{D}$ la mesure de Lebesgue restreinte à $D$ et normalisée pour que sa masse totale soit 1 . On note $\delta(B, D)=\delta\left(\mu_{B}, \mu_{D}\right)$.

Théorème 4. On suppose les liaisons attractives (i.e. $\left.e\left(a_{1}, a_{2}\right) \geq 0\right)$ et à courte portée $\left(e\left(a_{1}, a_{2}\right)=0\right.$ si la distance de $a_{1} \grave{a} a_{2}$ est trop grande). Pour $N$ entier, soit $B_{N}$ une partie finie de l'ensemble $A$ des atomes qui minimise l'énergie de surface parmi les parties à $N$ éléments. On suppose que le centre de gravité de $B_{N}$ est à 
l'origine. Il existe pour chaque $N$ un homothétique $B_{N}^{\prime}$ de $B_{N}$ tel que, lorsque $N$ tend vers l'infini, la suite $B_{N}^{\prime}$ converge pour la distance $\delta$ vers la boule unité de la norme cristalline.

En d'autres termes, en un sens faible, les cristaux réels convergent vers un polyèdre convexe. Celui-ci peut être de dimension inférieure à 3. Cela décrit des cristaux lamellaires ou aciculaires (en forme d'aiguilles). Les hypothèses sur les liaisons sont très restrictives. Elles excluent les cristaux ioniques, mais s'appliquent aux liaisons covalentes.

Le polyèdre convexe obtenu minimise à volume constant une énergie de surface. Voici comment retrouver la tension superficielle correspondante. Soit $P$ un plan, $w$ un vecteur unitaire normal à $P$. Notons $\ell$ la forme linéaire $x \mapsto \ell(x)=\langle w, x\rangle$. Considérons les plans parallèles $P_{t}=\{\ell(x)=t\}$. Le nombre (la mesure) des plans de cette famille qui coupent un segment $\left[a_{1}, a_{2}\right]$ est $\left|\ell\left(a_{2}\right)-\ell\left(a_{1}\right)\right|$. Par conséquent, le nombre

$$
\sum_{\left(a_{1}, a_{2}\right) \in A \times A / G} e\left(a_{1}, a_{2}\right)\left|\ell\left(a_{2}\right)-\ell\left(a_{1}\right)\right|
$$

représente l'énergie totale par cellule des liaisons coupées en moyenne par $P$ et ses translatés. Ce nombre dépend de la position des atomes dans la cellule, alors que la forme d'équilibre du cristal ne devrait dépendre que des énergies. Déplaçons les atomes dans la cellule en conservant la périodicité par le groupe $G$. Cela revient à remplacer chaque atomes $a$ par $a+\delta(a)$ où le vecteur $\delta(a)$ est fonction périodique de $a$. L'expression $\ell\left(a_{2}\right)-\ell\left(a_{1}\right)$ est changée en $f\left(a_{2}\right)-f\left(a_{1}\right)$ où $f=\ell+\ell \circ \delta$ est un cocycle représentant $\ell$. Inversement, tout cocycle représentant $\ell$ est de cette forme. Par conséquent, la borne inférieure figurant dans la définition 4 est l'énergie moyenne des liaisons coupées par les plans parallèles à $P$, rendue insensible aux fluctuations périodiques des atomes. La tension superficielle $\nu(P)$ dans la direction de $P$ est l'inverse de ce nombre.

Une fois calculée la tension superficielle, la forme d'équilibre s'obtient par la construction de Wulff classique. Pour chaque plan $P$, on considère le demi-espace contenant l'origine, bordé par le plan parallèle à $P$ situé à distance $\nu(P)$ de l'origine. La forme d'équilibre est l'intersection de ces demi-espaces.

Exemple : Le diamant. Il possède deux atomes et quatre liaisons par cellule. Les liaisons, qui ont toutes même énergie, relient un atome à ses quatre plus proches voisins qui forment un tétraèdre régulier. La boule unité de la norme cristalline est homothétique à l'octaèdre régulier obtenu comme enveloppe convexe des milieux des côtés de ce tétraèdre. Le calcul est détaillé en section 13.4.

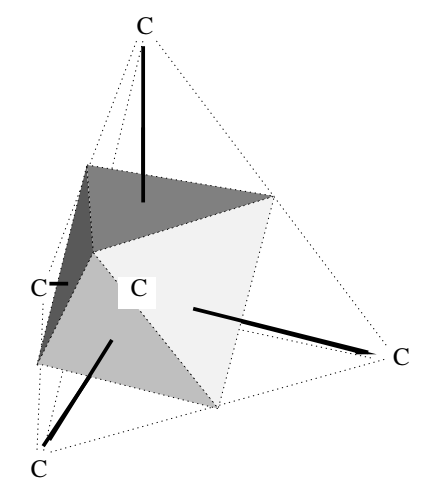

\subsection{Discussion}

Il y a d'autres modèles déterministes pour les cristaux. Par exemple, Wills [33] adopte le point de vue des empilements de sphères dures (voir aussi [30]). Il considère un réseau $A$ de $\mathbb{R}^{3}$ et des parties finies $B$ de $A$ d'un type spécial : les intersections de $A$ avec des polyèdres convexes rationnels. Il étudie une famille de principes variationnels dépendant d'un paramètre $\rho$ : il s'agit de maximiser la $\rho$-densité de $B$, définie comme l'inverse du volume de l'enveloppe convexe du voisinage tubulaire de rayon $\rho$ de $B$. Wills montre que, lorsque $B=A \cap K$ est grand, cela revient asymptotiquement à minimiser l'énergie de surface de $K$ pour une certaine tension superficielle (qui dépend de $\rho$ ), donc les parties minimisantes convergent vers une unique forme optimale (pour chaque valeur de $\rho$ ). 
Ce modèle diffère de celui du théorème 4 en ce que l'énergie des liaisons est imposée par la géométrie de la maille.

La faiblesse du théorème 4 , comme pour tous les modèles déterministes, est l'absence du paramètre température, dont la tension superficielle dépend et qui est à l'origine des phénomènes les plus intéressants (transitions de phases, ...). En dimension 2, pour les modèles d'Ising [12], de percolation de Bernoulli [1] et solide/solide en dimension $1+1$ [11], on sait valider la construction de Wulff pour une température non nulle mais suffisamment basse. Toutefois, dans les deux premiers résultats, l'énergie des liaisons est la plus simple possible : 1 entre 2 sites voisins sur le réseau $\mathbb{Z}^{2}, 0$ sinon. Le problème en température nulle est élémentaire dans ce cas.

En revanche, le théorème 4 semble un préliminaire nécessaire à l'étude des modèles probabilistes en dimension supérieure. En effet, Dobrushin et al., à la fin de l'introduction de [12], évoquent la généralisation de leurs résultats sur le modèle d'Ising en dimension 2 aux dimensions supérieures et diagnostiquent la principale difficulté : pour eux, c'est la technique liée aux topologies sur les espaces de domaines. Toutefois, la technique choisie dans le présent papier (courants) n'est pas la seule possible, et Cerf [10] a pu traiter le modèle de percolation de Bernoulli en dimension 3 en utilisant les ensembles de périmètre borné.

\subsection{La preuve}

Elle consiste à comparer du point de vue isopérimétrique deux métriques sur $\mathbb{R}^{n}$, la métrique riemannienne périodique donnée et une norme non euclidienne (il s'agit de la norme $|\cdot|_{\infty} \operatorname{sur} H^{n-1}(T, \mathbb{R})=\mathbb{R}^{n}$ ). Dans un premier temps, on précise en section 2 la définition adaptée du volume pour les hypersurfaces dans un espace vectoriel normé, ou plus généralement dans une variété munie d'une métrique finslérienne.

En utilisant le théorème de compacité pour les courants et une propriété de semi-continuité du volume (prouvée en Sect. 6), on montre en section 7 que les bords des grands domaines ont un plus grand volume pour la métrique périodique que pour la norme $|\cdot|_{\infty}$.

L'inégalité inverse s'obtient en construisant de grands domaines dont le volume riemannien approche le volume au sens de la norme $|\cdot|_{\infty}$. Il s'avère plus commode dans un premier temps de comparer des fonctions (voir en Sect. 8). Autrement dit, de travailler sur l'inégalité de Sobolev plutôt que sur l'inégalité isopérimétrique (Sect. 4).

Pour compléter la preuve du théorème 1, il suffit de connaître l'inégalité isopérimétrique dans un espace normé. Ce résultat remonte à la thèse de Brunn en 1887 (Sect. 9). Le théorème 2 résulte simplement de l'inégalité de Hölder. Les exemples de la proposition 3 sont présentés en section 11. Ce sont les mêmes que ceux de $[6]$.

Le théorème 3 s'obtient en combinant le théorème 1 avec l'inégalité de Blaschke-Santalo (qui permet de comparer les volumes asymptotiques d'un réseau et de son dual) et l'inégalité de Burago-Ivanov sur le volume asymptotique des tores, voir section 10.

Pour le cas particulier $n=2$, il existe une preuve plus simple [27].

Le théorème 4 s'obtient en appliquant le théorème 1 à des métriques riemanniennes particulières, qui présentent une concentration de volume au voisinage des atomes et qui donnent une grande aire aux surfaces qui coupent les liaisons (Sects. 12 et 13).

\subsection{Questions}

Le volume conforme $V A\left(H^{n-1}(T, \mathbb{Z}),|\cdot|_{n / n-1}\right)$ est il la borne supérieure de $\left(c_{\infty} / n\right)^{n}$ (resp. la borne inférieure du volume asymptotique du revêtement universel) dans une classe conforme ?

Peut on affiner le développement asymptotique du théorème 1 ? La preuve donne la convergence en topologie $b$ des domaines extrémaux (qui minimisent le volume du bord à volume donné pour la métrique périodique) vers les "gouttes", i.e. les domaines extrémaux pour la norme $|\cdot|_{\infty}$. Peut on préciser cette convergence?

Le problème isopérimétrique asymptotique peut se formuler en termes d'inégalité de Sobolev $L_{1}^{1} \subset L^{n / n-1}$, voir en section 43. Quel est le comportement des fonctions extrémales pour l'inégalité de Sobolev $L_{1}^{p} \subset L^{n p / n-p}$ 
avec $p>1$ ? Il est tentant d'appliquer les méthodes de la théorie de l'homogénéïsation, voir par exemple [31] chapitre 5. Lorsque $p=2$, la question est voisine de celle du comportement asymptotique du noyau de la chaleur en temps grand, examiné dans [20].

Soit $S$ un système générateur fini de $\mathbb{Z}^{n}$. Etant donnée une partie finie $D$ de $\mathbb{Z}^{n}$, on définit le bord $\partial D$ comme l'ensemble des points $e \in D$ tel que $e S$ n'est pas contenu dans $D$. On obtient ainsi un profil isopérimétrique $I_{S}: \mathbf{N} \rightarrow \mathbf{N}$. Est-il vrai que $I_{S}(\tau) / \tau^{n-1 / n}$ converge ? Cela ne semble pas résulter du théorème 4 .

Le cadre naturel du problème isopérimétrique asymptotique est celui des variétés riemanniennes munies d'une action isométrique et cocompacte d'un groupe discret $\Gamma$ à croissance polynômiale. Même lorsque $\Gamma=\mathbb{Z}^{n}$, le théorème 1 ne porte que sur un cas particulier.

\section{VOLUME DES HYPERSURFACES DANS LES VARIÉTÉS FINSLÉRIENNES}

\subsection{Terminologie des courants}

Soit $V$ une variété riemannienne de dimension $n$. Un courant de dimension $k$ sur $V$ est une fonctionnelle sur l'espace des $k$-formes différentielles lisses à support compact. Le bord $\partial C$ d'un courant $C$ est défini par $\partial C(\phi)=C(d \phi)$. Si $f: V \rightarrow W$ est une application de classe $C^{\infty}$, le courant $f_{\sharp} C$ est défini par $f_{\sharp} C(\phi)=C\left(f^{*} \phi\right)$.

Étant donnée une norme sur $\Lambda^{k} T^{*} V$ dépendant continument du point, on définit la masse d'un courant de dimension $k$ comme la borne supérieure de ses valeurs sur les formes différentielles de norme $L^{\infty}$ plus petite que 1. Un courant est dit normal si $C$ et $\partial C$ sont à support compact et de masse finie (cela ne dépend pas de la norme particulière choisie sur $\left.\Lambda^{*} T^{*} V\right)$. La norme b d'un courant $C$, notée $\|C\|_{b}$ est la borne inférieure des nombres $M(R)+M(S)$ sur les courant $R$ et $S$ tels que $C=R+\partial S$.

Exemple. Soit $W$ une sous-variété à bord de $V$, compacte, de classe $C^{1}$, orientée. Alors $W$ définit un courant d'intégration $I_{W}$. On a $\partial I_{W}=I_{\partial W}$. Si $V$ est riemannienne et si on utilise la norme comasse sur l'algèbre extérieure (voir [14], p. 38), la masse de $I_{W}$ est égale au volume de $W$.

\subsection{Courants étalés et intégrand de Poincaré}

On suppose $V$ orientée. Soit $\alpha$ une $n-k$-forme différentielle $L_{l o c}^{1}$ à support compact sur $V$. On note $C_{\alpha}$ le courant de dimension $k$

$$
C_{\alpha}(\phi)=\int_{V} \alpha \wedge \phi
$$

Si de plus $d \alpha \in L_{l o c}^{1}$, alors le courant $C_{\alpha}$ est normal. On l'appelle courant étalé associé à $\alpha$. Sa masse ne s'exprime simplement que pour un choix judicieux de normes sur $\Lambda^{k} T^{*} V, \Lambda^{n-k} T^{*} V$ et $\Lambda^{n} T^{*} V$. Noter que la donnée d'une orientation et d'une norme sur $\Lambda^{n} T^{*} V$ équivaut à celle d'un élément de volume, i.e. d'une $n$-forme continue, qui ne s'annulle pas, et qui est compatible avec l'orientation.

Définition 6. Soit $R$ un espace vectoriel de dimension $n$ muni d'un élément de volume $\omega$. Des normes sur $\Lambda^{k} R^{*}$ et $\Lambda^{n-k} R^{*}$ sont dites Poincaré-duales si

$$
|\phi|=\sup \left\{\alpha \wedge \phi / \omega ; \alpha \in \Lambda^{n-k} R^{*},|\alpha| \leq 1\right\} .
$$

Si $R$ est muni d'une norme, on appelle intégrand de Poincaré la norme sur $\Lambda^{n-1} R^{*}$ Poincaré-duale de la norme duale sur $R^{*}$.

Désormais, quand on parlera de profil isopérimétrique pour un espace vectoriel normé ou plus généralement une variété finslérienne, c'est qu'un élément de volume est spécifié (pour définir le volume des domaines) et qu'on utilise l'intégrand de Poincaré (pour définir le volume de leurs bords).

Par construction, avec ce choix de normes, la masse d'un courant étalé coïncide avec la norme $L^{1}$. 
Lemme 7. Si une variété $V$ est équipée d'un élément de volume $\omega$ et de normes Poincaré-duales sur $\Lambda^{k} T^{*} V^{*}$ et $\Lambda^{n-k} T^{*} V^{*}$, alors pour toute $k$-forme différentielle $\alpha$ sur $V$

$$
M\left(C_{\alpha}\right)=\int_{V}|\alpha| \omega .
$$

Remarque. Lorsque $R$ est muni d'une norme euclidienne, les normes comasses en dimension $k$ et $n-k$ sont Poincaré-duales si et seulement si $k \leq 1$ ou $k \geq n-1$.

\subsection{Le problème isopérimétrique asymptotique}

Soit $T$ un tore finslérien orienté. On note $E=H_{1}(T, \mathbb{Z}), R=H_{1}(T, \mathbb{R})$ On munit l'espace vectoriel $R^{*}=H^{1}(T, \mathbb{R})$ de la norme $|\cdot|_{1}$ quotient de la norme $L^{1}$ sur les 1 -formes différentielles fermées, et on munit $R$ de la norme duale. Le tore $T_{\infty}=R / E$ devient à son tour une variété finslérienne. À homotopie près, il y a une unique équivalence d'homotopie $: T \rightarrow T_{\infty}$ induisant l'identité sur $H_{1}$. Cela permet d'identifier canoniquement la cohomologie de $T_{\infty}$ à celle de $T$. En particulier, l'orientation de $T$ détermine une orientation de $T_{\infty}$.

Définition 8. On note $\omega_{\infty}$ l'élément de volume invariant par translation sur $T_{\infty}$ dont l'intégrale vaut 1 . La masse limite $M_{\infty}$ est la masse induite sur les courants sur $T_{\infty}$ par l'intégrand de Poincaré correspondant. Enfin, on note $I_{\infty}$ le profil isopérimétrique de l'espace vectoriel normé $R$.

Le théorème 1 est un cas particulier de l'énoncé suivant, qui sera démontré en section 9.

Proposition 9. Soit $T$ un tore de dimension $n$ muni d'une métrique finslérienne, d'un élément de volume $\omega$ et de l'intégrand de Poincaré correspondant. On note I le profil isopérimétrique du revêtement universel. Alors

$$
\lim _{\tau \rightarrow+\infty} I(\tau) / I_{\infty}(\tau)=\left(\int_{T} \omega\right)^{1-n / n} .
$$

\section{PropriÉtÉS DES COURANTS}

\subsection{Courants entiers}

Définition 10. Une chaîne (resp. entière) est une combinaison linéaire finie à coefficients réels (resp. entiers) de courants d'intégration sur des sous-variétés compactes à bord orientées. Les chaînes sont denses dans les courants normaux pour la topologie b. Un courant est dit entier (en anglais, integral current) s'il est normal et dans l'adhérence pour la topologie $b$ des chaînes entières.

Plus précisément

Proposition 11. ([14], p. 417). Soit $C$ un courant normal (resp. entier) et $\epsilon>0$. Il existe une chaîne $\tilde{C} \grave{a}$ coefficients réels (resp. entiers) à support dans le $\epsilon$-voisinage du support de $C$ et telle que $M(\tilde{C}-C)+M(\partial(\tilde{C}-$ $C))<\epsilon$.

L'intérêt de la théorie des courants pour notre problème isopérimétrique asymptotique tient dans le théorème de compacité suivant.

Proposition 12. ([14], p. 414). Soit $K$ un compact de $V$. L'ensemble des courants entiers $C$ à support dans $K$ et tels que $M(C)+M(\partial C) \leq$ const. est compact pour la topologie b.

\subsection{Formule de la coaire}

La formule de la coaire relie certains courants étalés aux courants d'intégration. 
Proposition 13. ([14], p. 248 et p. 438). Soit u une fonction lisse sur $V$. Alors pour presque tout $s \in \mathbb{R}$, $\{u=s\}$ est une sous-variété et

$$
C_{d u}=\int_{\mathbb{R}} I_{\{u=s\}} d s .
$$

En particulier, si les normes sur $T^{*} V$ et sur $\Lambda^{n-1} T^{*} V$ sont Poincaré-duales,

$$
\int_{V}|d u| \omega=\int_{\mathbb{R}} M\left(I_{\{u=s\}}\right) d s .
$$

\section{InÉGAlité DE SOBOLEV}

\subsection{Approximation par des courants étalés}

Soit $V$ une variété de dimension $n$ munie d'un élément de volume. Tout courant normal de dimension $n$ est étalé, i.e. de la forme $C_{u}$ où $u$ est une fonction $L^{1}$ à support compact. En effet, c'est vrai pour les chaînes à coefficients réels donc pour leur adhérence pour la norme b (qui coïncide avec la masse ou la norme $L^{1}$ dans cette dimension). Par conséquent tout courant normal de dimension $n$ est limite de courants de la forme $C_{u_{\epsilon}}$ avec $u_{\epsilon}$ lisse. Nous aurons besoin que $u_{\epsilon}$ converge dans d'autres normes que $L^{1}$. Cela nécessite une construction particulière.

Lemme 14. Soit $V$ une variété dimension $n$ munie d'un élément de volume et d'une norme sur $\Lambda^{n-1} T^{*} V$. Soit $C=C_{u}$ un courant normal. Pour tout $\epsilon>0$ il existe une fonction lisse $u_{\epsilon}$ telle que $M\left(\partial C_{u_{\epsilon}}\right)<M(\partial C)+\epsilon$ et le support de $u-u_{\epsilon}$ a un volume $<\epsilon$.

Preuve. On munit $T^{*} V$ de la norme Poincaré-duale de celle de $\Lambda^{n-1} T^{*} V$, puis $T V$ de la norme duale de celle de $T^{*} V$. Soit $W \subset V$ une sous-variété compacte à bord de classe $C^{\infty}$. Notons $\rho$ la fonction qui, hors de $W$ (resp. dans $W$ ) coïncide avec la distance finslérienne au bord de $W$ (resp. son opposé). Cette fonction est $C^{\infty}$ au voisinage de $\partial W$ et satisfait $|d \rho|=1$. On fixe $\epsilon>0$ et on pose $u_{\epsilon, W}=1-\rho / \epsilon$ sur le $\epsilon$-voisinage $U$ de $\partial W$, $u_{\epsilon, W}=1$ sur $W \backslash U$ et $u_{\epsilon, W}=0$ hors de $W \cup U$. Lorsque $\epsilon$ tend vers $0, u=u_{\epsilon, W}$ hors du $\epsilon$-voisinage $U$, dont le volume est < const. $\epsilon$.

Soit $C$ un courant normal. On l'approche d'abord par une chaîne $\sum \lambda_{i} I_{W_{i}}$ à coefficients réels, proposition 11. Il ne coûte rien de supposer les $W_{i}$ de classe $C^{\infty}$ et deux à deux transverses. On pose $u_{\epsilon}=\sum_{i} \lambda_{i} C_{u_{\epsilon}, W_{i}}$. Alors $M\left(C_{u_{\epsilon}}-C\right)$ tend vers 0 . Quitte à retirer de petits voisinages des objets de codimension au moins 2 (intersections $\partial W_{i} \cap \partial W_{j}$ ) où la masse de $\partial C$ comme celle de $C_{d u_{\epsilon}}$ est petite, on peut supposer que $\partial C$ est la somme de courants d'intégration à supports disjoints,

$$
\partial C=\sum \lambda_{\mathbf{i}, j} I_{W_{\mathbf{i}, j}} \quad \text { où } \quad W_{i_{1}, \ldots, i_{\ell}, j}=W_{i_{1}} \cap \cdot \cap W_{i_{\ell}} \cap \partial W_{j} .
$$

La masse de $\partial C$ est la somme des masses. Au voisinage de $W_{\mathbf{i}, j}, d u_{\epsilon}=\lambda_{j} d u_{\epsilon, W_{j}}$. D'après la formule de la coaire (Prop. 13), le volume du voisinage tubulaire de $W_{\mathbf{i}, j}$ de largeur $\epsilon$ est équivalent à $\epsilon M\left(W_{\mathbf{i}, j}\right)$. On conclut que $\int\left|d u_{\epsilon}\right| \omega$ converge vers $M(\partial C)$.

Enfin, il ne coûte rien d'approcher $u_{\epsilon}$ par une fonction lisse.

Remarque 15. Soit $C=C_{u}$ un courant normal de dimension $n$. On dit que $C$ est positif si $u \geq 0$. On appelle le courant $|C|=C_{|u|}$ sa valeur absolue. Clairement, $M(|C|)=M(C)$. En utilisant le lemme 14, on montre aisément que $M(\partial|C|) \leq M(\partial C)$.

Par conséquent, pour le problème isopérimétrique, on peut se limiter aux courants positifs. 


\subsection{Inégalité isopérimétrique et courants}

On peut modifier la définition du profil isopérimétrique en élargissant la classe des domaines (sous-variétés compactes à bord de codimension 0) à celle des courants entiers (domaines "avec multiplicités"). Dans ce paragraphe, on vérifie que pour un espace vectoriel muni d'un élément de volume et d'une norme sur les $n$-1-formes, cela ne change pas le profil.

Proposition 16. Soit $V$ une variété finslérienne orientée munie d'un élément de volume $\omega$. On munit $\Lambda^{n-1} T^{*} V$ de la norme Poincaré-duale de la norme duale naturelle de $T^{*} V$. Alors les propriétés suivantes sont équivalentes.

1. Pour tout domaine $D$ de $V, \int_{D} \omega \leq c(M(\partial D))^{n / n-1}$;

2. pour toute fonction u lisse à support compact sur $V$,

$$
\int|u|^{n / n-1} \omega \leq c\left(\int|d u| \omega\right)^{n / n-1}
$$

3. pour tout courant entier $C$ sur $V$ de dimension $n$,

$$
M(C) \leq c(M(\partial C))^{n / n-1} .
$$

Preuve. $\quad$ La preuve de $(1) \Leftrightarrow(2)$ est classique dans le cas riemannien $[13,19,21]$.

Soit $u$ une fonction lisse à support compact sur $V$. Comme $|d| u|| \leq|d u|$, on peut supposer $u \geq 0$. Pour $t>0$, notons $\chi_{t}$ la fonction caractéristique de l'ensemble de niveau $\{u>t\}$. Pour tout point $x \in V$,

$$
u(x)=\int_{0}^{+\infty} \chi_{t}(x) d t .
$$

Il vient

$$
\|u\|_{L^{n / n-1}} \leq \int_{0}^{+\infty}\left\|\chi_{t}\right\|_{L^{n / n-1}} d t=\int_{0}^{+\infty}\left(\int_{\{u>t\}} \omega\right)^{n / n-1} d t \leq c^{1-n / n} \int_{0}^{+\infty} M\{u=t\} d t=\int|d u| \omega .
$$

Inversement, étant donné un domaine lisse $D$, on applique l'inégalité de Sobolev à une approximation de la fonction caractéristique de $D$ et on obtient l'inégalité isopérimétrique, grâce à la formule de la coaire (Prop. 13).

Il reste à montrer que (2) entraîne (3). Soit $C$ un courant entier de dimension $n$. Grâce au lemme 14, on approche $C=C_{u}$ par le courant étalé $C_{u_{\epsilon}}$ associé à une fonction lisse $u_{\epsilon}$. L'inégalité de Sobolev donne

$$
\left(\frac{1}{c} \int_{V}\left|u_{\epsilon}\right|^{n / n-1} \omega\right)^{n-1 / n} \leq M\left(C_{d u_{\epsilon}}\right) \leq M(\partial C)+\epsilon .
$$

Lorsque $\epsilon$ tend vers $0, u_{\epsilon}$ ne diffère de $u$ que sur un ensemble dont le volume tend vers 0 . Par conséquent $\left\|u_{\epsilon}\right\|_{L^{n / n-1}}$ tend vers $\|u\|_{L^{n / n-1}}$ qui est $\geq M(C)^{n-1 / n}$ car $u$ est à valeurs entières.

\section{Calcul de la masse limite $M_{\infty}$}

\subsection{Comparaison de normes}

Dans ce paragraphe, on relie l'intégrand de Poincaré de $T_{\infty}$ aux normes naturelles sur $H_{n-1}(T, \mathbb{R})$ et $H^{n-1}(T, \mathbb{R})$.

Soit $V$ une variété compacte orientée de dimension $n$, munie d'un élément de volume et de de normes Poincaré-duales sur $\Lambda^{*} T^{*} V$. On note $|\cdot|_{\mathbb{R}}$ la norme sur $H_{k}(V, \mathbb{R})$ quotient de la masse des courants, on note $|\cdot|_{1}$ et $|\cdot|_{\infty}$ les normes sur $H^{k}(V, \mathbb{R})$ quotient des normes $L^{1}$ et $L^{\infty}$ sur les $k$-formes différentielles fermées. 
Lemme 17. Soit $V$ une variété compacte orientée de dimension n, munie d'un élément de volume et de de normes Poincaré-duales sur $\Lambda^{*} T^{*} V$. Le théorème des coefficients universels réalise $\left(H^{k}(V, \mathbb{R}),|\cdot|_{\infty}\right)$ comme le dual de $\left(H_{k}(V, \mathbb{R}),|\cdot|_{\mathbb{R}}\right)$. La dualité de Poincaré $\left(H^{k}(V, \mathbb{R}),|\cdot|_{1}\right) \rightarrow\left(H_{n-k}(V, \mathbb{R}),|\cdot|_{\mathbb{R}}\right)$ est une isométrie.

Preuve. Elle est tirée essentiellement de [17] chapitre 4.

Soit $R$ un espace vectoriel normé orienté de dimension $n$ muni d'un élément de volume $\omega$. On munit $\Lambda^{*} R^{*}$ de normes Poincaré-duales. Alors la forme bilinéaire

$$
\Lambda^{k} R \otimes \Lambda^{n-k} R \rightarrow \mathbb{R}, \quad \alpha, \phi \mapsto \alpha \wedge \phi / \omega
$$

met les espaces vectoriels normés $\Lambda^{k} R^{*}$ et $\Lambda^{n-k} R^{*}$ en dualité isométrique.

La dualité de Poincaré est la forme bilinéaire qui à une $k$-forme différentielle $\alpha$ sur $V$ et une $n-p$-forme différentielle $\phi$ associe $\int_{V} \alpha \wedge \phi$. Lorsque $\Lambda^{*} T^{*} V$ est muni de normes Poincaré-duales, elle réalise l'espace des $k$-formes $L^{1}$ comme le dual de l'espace des $k$-formes $L^{\infty}$.

Cette propriété passe au quotient. En effet, soit $\alpha$ une $k$-forme fermée telle que $\|\alpha\|_{L^{1}}=1,[\alpha]$ sa classe de cohomologie. Montrons qu'il existe une $n-k$-forme fermée $\phi$ telle que $\|\phi\|_{L^{\infty}}=1$ et $\int \alpha \wedge \phi=1$. Il existe une forme linéaire $L$ sur $H^{k}(V, \mathbb{R})$ telle que

$$
\sup \left\{L([\beta]) ;[\beta] \in H^{p}(V, \mathbb{R}),\|\alpha\|_{L^{1}}=1\right\}=1, \text { atteint en } \quad \alpha .
$$

$L$ définit donc une forme linéaire continue sur l'espace des $k$-formes fermées $L^{1}$, de norme 1 , nulle sur les formes exactes. Par Hahn-Banach, $L$ admet un prolongement en une forme linéaire continue sur l'espace des $k$-formes fermées $L^{1}$, de norme 1 , nulle sur les formes exactes. Comme $L^{\infty}$ est le dual de $L^{1}$, cette forme linéaire est donnée par l'intégration contre une $n$-1-forme $\phi$. Nécessairement, $\|\phi\|_{L^{\infty}}=\|L\|=1$. Pour toute $k-1$-forme $\gamma$ de classe $C^{1}, \int d \gamma \wedge \phi=0, \phi$ est fermée. Enfin, $\int \alpha \wedge \phi=L(\alpha)=1$. Ceci prouve que la norme quotient $|\cdot|_{\infty}$ sur $H^{n-k}(V, \mathbb{R})$ est la norme duale de $|\cdot|_{1}$ sur $H^{k}(V, \mathbb{R})$.

Le même argument s'applique à la forme bilinéaire qui à une $n-k$-forme différentielle $\phi$ et un $n-k$-courant $C$ associe $C(\phi)$. Elle réalise l'espace des $n-k$-courants de masse finie comme le dual de l'espace des $n-k$-formes continues muni de la norme $L^{\infty}$ (borne supérieure des normes comasse ponctuelles). Par conséquent, la norme quotient $|\cdot|_{\infty} \operatorname{sur} H^{n-k}(V, \mathbb{R})$ est la norme duale de $|\cdot|_{\mathbb{R}}$, quotient de la masse, sur $H_{n-k}(V, \mathbb{R})$.

On conclut que $\left(H^{k}(V, \mathbb{R}),|\cdot|_{1}\right)$ et $\left(H_{n-k}(V, \mathbb{R}),|\cdot|_{\mathbb{R}}\right)$ sont isométriques.

Remarque. Voici une preuve plus concrète. L'injection isométrique $\alpha \mapsto C_{\alpha}$ donne précisément la dualité de Poincaré, par passage au quotient. Inversement, une variante du lemme 14 permet d'approcher tout $n-1$ courant normal fermé $C$ par un courant étalé $C_{\alpha}$ où $\alpha$ est une 1-forme fermée lisse sur $V$. Par construction, $C_{\alpha}$ est cohomologue à $C$ et de masse voisine de celle de $C$. Ceci montre que la dualité de Poincaré est une isométrie.

Remarque. Comme signalé en 7 , la proposition 17 ne s'applique aux variétés riemanniennes que pour $k \leq 1$ et $k \geq n-1$.

Corollaire 18. Soit $T$ un tore finslérien muni d'un élément de volume et de l'intégrand de Poincaré correspondant. On note $R=H_{1}(V, \mathbb{R})$, muni de la norme duale de $|\cdot|_{1}$. On note

$$
F: \Lambda^{k} R^{*} \rightarrow H^{k}\left(T_{\infty}, \mathbb{R}\right) \rightarrow H^{k}(T, \mathbb{R})
$$

l'isomorphisme qui envoie un covecteur vu comme forme différentielle à coefficients constants sur $T_{\infty}$ sur sa classe de cohomologie. Alors l'intégrand de Poincaré sur $\Lambda^{n-1} T^{*} T_{\infty}=\Lambda^{n-1} R^{*}$ s'obtient en transportant par cet isomorphisme la norme quotient de la norme $L^{\infty}$ sur les $k$-formes différentielles fermées sur $T$. 
Preuve. Par transport par l'isomorphisme $F$ de la classe fondamentale en cohomologie du tore orienté $T$, on dispose d'une norme sur $\Lambda^{n} R^{*}$. Par définition, l'intégrand de Poincaré est la norme sur $\Lambda^{n-1} R^{*}$ donnée par

$$
|\psi|=\sup \left\{|\beta \wedge \psi| ; \beta \in R^{*},|\beta|_{1} \leq 1\right\}
$$

Par construction, l'isomorphisme $F$ transporte le produit extérieur $R^{*} \otimes \Lambda^{n-1} R^{*} \rightarrow \Lambda^{n} R^{*}$ sur le cup-produit $H^{1}(T, \mathbb{R}) \otimes H^{n-1}(T, \mathbb{R}) \rightarrow H^{n}(T, \mathbb{R})$. Par conséquent, si $\alpha$ est un représentant de la classe $F(\beta)$ et $\phi$ est un représentant de la classe $F(\psi)$, on a

$$
|\beta \wedge \psi|=\int_{T} \alpha \wedge \phi
$$

et

$$
|\psi|=\sup \left\{\int_{T} \alpha \wedge \phi ; \alpha 1-\text { forme fermée, }\|\alpha\|_{L^{1}} \leq 1\right\}=\inf \left\{\|\phi\|_{L^{\infty}} ; \phi \in \psi\right\}
$$

d'après le lemme 17.

\subsection{Courants et formes minimisants}

Lemme 19. Soit $R$ un espace vectoriel normé orienté, $E \subset R$ un réseau. On muni $R$ de l'élément de volume d'intégrale 1 sur $R / E$ et de l'intégrand de Poincaré associé. Soit $P$ un parallélépipède dans $R$ formé sur des vecteurs entiers. On note $\pi(P)$ son image dans $R / E$. Alors le courant d'intégration $I_{\pi(P)}$ a une masse minimum dans sa classe d'homologie réelle, i.e. réalise la norme $\left|\left[I_{\pi(P)}\right]\right|_{\mathbb{R}}$. De même, toute $n-1$-forme invariante par translations sur $R / E$ minimise la norme $L^{\infty}$ dans sa classe de cohomologie.

Preuve. Soit $P$ le parallélépipède formé sur $n-1$ vecteurs entiers linéairement indépendants $v_{1}, \ldots, v_{n-1} \in E$. Le courant d'intégration sur l'image de $P$ dans $R / E$ consiste à intégrer sur

$$
\sigma_{P}:(\mathbb{R} / \mathbb{Z})^{n-1} \rightarrow R / E, \quad\left(s_{1}, \ldots, s_{n-1}\right) \mapsto \sum s_{j} v_{j}
$$

Montrons que ce courant minimise la masse dans sa classe d'homologie réelle. Notons $\xi_{P}=v_{1} \wedge \cdots \wedge v_{n-1}$ $\in \Lambda_{n-1} H_{1}(T, \mathbb{R})$. Munissons $\Lambda^{n-1} R$ de la norme $|\cdot|^{*}$ duale de l'intégrand de Poincaré. Par Hahn-Banach, il existe une forme linéaire $\phi \in \Lambda^{n-1} R^{*}$ qui vaut 1 sur $\xi_{P} /\left|\xi_{P}\right|^{*}$ et prend des valeurs $\leq 1$ sur tout $n-1$-vecteur de norme $\leq 1$. On peut voir $\phi$ comme une forme différentielle invariante par translations (et donc fermée) sur $R / E$. Par construction, elle calibre $\sigma_{P}$. Par conséquent, $I_{\pi(P)}$ minimise la masse dans sa classe d'homologie réelle et

$$
\left|\left[I_{\pi(P)}\right]\right|_{\mathbb{R}}=M\left(I_{\pi(P)}\right)=\int_{\sigma_{P}} \phi=\phi\left(\xi_{P}\right)=\left|\xi_{P}\right|^{*} .
$$

Si $\psi$ est une $n-1$-forme différentielle sur $T$ cohomologue à $\phi$, alors

$$
\phi\left(\xi_{P}\right)=C(\phi)=C(\psi)=\int_{(\mathbb{R} / \mathbb{Z})^{n-1}} \psi_{\sigma_{P}\left(t_{1}, \ldots, t_{n-1}\right)}\left(\xi_{P}\right)
$$

donc il existe un point $x=\sigma_{P}\left(t_{1}, \ldots, t_{n-1}\right)$ de $R / E$ tel que $\psi_{x}\left(\xi_{P}\right) \geq \phi\left(\xi_{P}\right)$. Par conséquent, $\|\phi\|_{L^{\infty}}$ $\leq\|\psi\|_{L^{\infty}}$. Ceci montre que $\psi$ minimise la norme $L^{\infty}$ dans sa classe de cohomologie. Lorsque les vecteurs $v_{1}, \ldots, v_{n-1}$ varient, les formes linéaires $\phi$ décrivent une partie dense de le sphère unité de $\Lambda^{n-1} R^{*}$, donc toutes les $n-1$-formes invariantes par translations sur $R / E$ minimisent la norme $L^{\infty}$ dans leur classe de cohomologie.

Corollaire 20. Soit $T$ un tore finslérien muni d'un élément de volume dont l'intégrale vaut 1. $T_{\infty}=H_{1}(T, \mathbb{R}) / H_{1}(T, \mathbb{Z})$ muni de la métrique et de l'élément de volume définis en 8. Munissons $T$ et $T_{\infty}$ des intégrands de Poincaré. Alors l'isomorphisme naturel $H_{n-1}(T, \mathbb{R}) \rightarrow H_{n-1}\left(T_{\infty}, \mathbb{R}\right)$ est une isométrie pour les normes masses $|\cdot| \mathbb{R}$. 
Preuve. Le lemme 19 montre que l'application

$$
\Lambda^{n-1} R^{*} \rightarrow H^{n-1}\left(T_{\infty}, \mathbb{R}\right)
$$

qui à un covecteur vu comme forme différentielle à coefficients constants sur $T_{\infty}$ sur sa classe de cohomologie est une isométrie. En composant avec l'isomorphisme naturel $H^{n-1}\left(T_{\infty}, \mathbb{R}\right) \rightarrow H^{n-1}(T, \mathbb{R})$, on obtient l'application $F$ qui est une isométrie d'après le corollaire 18. On conclut que l'isomorphisme naturel est une isométrie.

\section{SEMi-COntinuité}

\subsection{Position du problème}

Soit $T$ un tore finslérien muni d'un élément de volume $\omega$ d'intégrale 1 . On note $R=H_{1}(T, \mathbb{R}), E=H_{1}(T, \mathbb{Z})$, $T_{\infty}=R / E$. D'après [24], il existe un difféomorphisme $\psi: T \rightarrow T_{\infty}$ qui envoie $\omega$ sur $\omega_{\infty}$. Transportons par $\psi$ la métrique de $T$ et relevons la au revêtement universel $R$ de $T_{\infty}$. On obtient une métrique finslérienne $E$-périodique sur $R$. On note $\pi: R \rightarrow R / E$ la projection.

On considère des courants entiers $C$ de dimension $n-1$ sur $R$, on note $M_{t}(C)=t^{1-n} M\left(\left(\delta_{t}\right)_{\sharp} C\right)$. L'objectif de cette section est de donner une généralisation du résultat suivant.

Proposition 21. ([14], p. 519). Soit $V$ une variété munie d'une norme sur $\Lambda^{k} T^{*} V$. La masse $M$ est une fonction semi-continue sur l'espace des courants entiers muni de la topologie b.

On va montrer la semi-continuité des masses lorsque $t$ tend vers l'infini et $C$ converge pour la topologie $b$.

Proposition 22. Soit $t_{j}$ une suite tendant vers $+\infty$ et soit $C_{j}$ une suite de courants entiers sur $R$, qui converge au sens de la topologie b vers $C$. Alors

$$
M_{\infty}(C) \leq \liminf M_{t_{j}}\left(C_{j}\right)
$$

\subsection{Courants $(k, \epsilon)$-rationnels}

On démontre d'abord la proposition dans le cas particulier où $C$ est un courant rationnel. Soit $R$ un espace vectoriel de dimension finie. On appelle subdivision polyédrale de $R$ un recouvrement fini de $R$ par des polyèdres convexes dont les intérieurs sont disjoints. On s'en sert pour découper les chaînes en morceaux. Noter que si $W$ est une sous-variété orientée en position générale par rapport à la subdivision $R=\bigcup \Delta_{i}$, alors le courant d'intégration $I_{W \cap \Delta_{i}}$ est bien défini et on a

$$
I_{W}=\sum_{i} I_{W \cap \Delta_{i}}, \quad M\left(I_{W}\right)=\sum_{i} M\left(I_{W \cap \Delta_{i}}\right)
$$

Définition 23. Soit $R$ un espace vectoriel de dimension $n, E \subset R$ un réseau, $\pi: R \rightarrow R / E$ la projection. Soit $\epsilon>0$. Soit $k$ un entier, $k \geq 1$. Un courant $(k, \epsilon)$-rationnel sur $R$ est une chaîne $C=\sum \lambda_{j} I_{W_{j}}$ de dimension $n-1$ telle qu'il existe une subdivision polyédrale $\mathbb{R}^{n}=\bigcup_{i \in I} \Delta_{i}$ telle que

- les sous-variétés $W_{j}$ sont en position générale par rapport aux polyèdres $\Delta_{i}$;

- $I=I_{0} \cup I_{1}$, et $\sum_{i \in I_{0}} M\left(C \cap \Delta_{i}\right)<\epsilon$;

- si $i \in I_{1}, P_{i}=C \cap \Delta_{i}$ est un multiple du courant d'intégration associé à une application affine $\sigma_{i}$ : $[0,1]^{n-1} \rightarrow \mathbb{R}^{n}$ et $\partial\left(\pi \circ \delta_{k}\right)_{\sharp} P_{i}=0$.

Autrement dit, à une erreur de faible masse près, $C$ est fait de parallélépipèdes construits sur des vecteurs à composantes rationnelles.

Lemme 24. Soit $\epsilon>0$ assez petit. Soit $C$ un $n-1$-courant $(k, \epsilon)$-rationnel sur $\mathbb{R}^{n}$. Soit $C^{\prime}$ un $n-1$-courant tel que $\left\|C^{\prime}-C\right\|_{b}<\epsilon$. Alors pour tout multiple entier $t$ de $k$,

$$
M_{\infty}(C) \leq M_{t}\left(C^{\prime}\right)+\text { const. } \epsilon .
$$


Preuve. Soit $\mathbb{R}^{n}=\bigcup_{i \in I=I_{0} \cup I_{1}} \Delta_{i}$ une subdivision polyédrale adaptée à $C$. Pour $v \in \mathbb{R}^{n}$, notons $\Delta_{v, i}$ le translaté de $\Delta_{i}$ par $v$. L'hypothèse de transversalité contenue dans la définition des courants $\epsilon$-rationnels entraîne que les courants $C \cap \Delta_{v, i}$ varient continûment en topologie $b$ en fonction de $v$ lorsque $v$ est petit. Par conséquent, il existe $\eta>0$ tel que

$$
\sum_{i \in I}\left\|C^{\prime} \cap \Delta_{v, i}-C \cap \Delta_{v, i}\right\|_{b}<\epsilon .
$$

Soient $R$ et $S$ des courants tels que $C^{\prime}-C=R+\partial S$ et $M(R)+M(S)<\epsilon$.

On va estimer la masse (en moyenne) des intersections de $S$ par des plans parallèles. Pour cela, on utilise temporairement une métrique euclidienne sur $\mathbb{R}^{n}$. Soit $P$ un hyperplan de vecteur normal $\nu, P_{v}$ son translaté par un vecteur $v$. Par Fubini ([14], p. 438),

$$
M(S)=\int_{\mathbb{R}} M\left(S \cap P_{s \nu}\right) d s .
$$

Comme $P_{v}=P_{\langle v, \nu\rangle \nu}$

$$
\int_{|v|<\eta} M\left(S \cap P_{v}\right) d v=\int_{\mathbb{R}} d s \int_{|v|<\eta,\langle v, \nu\rangle=s} M\left(S \cap P_{s \nu}\right) d v \leq \text { const. } \eta^{n-1} \int_{\mathbb{R}} M\left(S \cap P_{s \nu}\right) d s \leq \text { const. } M(S) .
$$

Remplaçons l'hyperplan $P$ par la réunion (finie) des hyperplans qui portent les bords des polyèdres $\Delta_{i}$. Il vient

$$
\int_{|v|<\eta}\left(\sum_{i \in I} M\left(S \cap \partial \Delta_{v, i}\right)\right) d v \leq \text { const. } M(S) .
$$

On peut donc choisir un vecteur $v$ tel que $|v|<\eta$ et $\sum_{i \in I} M\left(S \cap \partial \Delta_{v, i}\right)<$ const. $\epsilon$. Ce vecteur est fixé désormais. Soient $R_{i}$ et $S_{i}$ des courants tels que

$$
C \cap \Delta_{v, i}-C \cap \Delta_{i}=R_{i}+\partial S_{i} \quad \text { et } \quad \sum_{i \in I} M\left(R_{i}\right)+M\left(S_{i}\right)<\epsilon .
$$

On écrit

d'où

$$
C^{\prime} \cap \Delta_{v, i}-C \cap \Delta_{v, i}=R \cap \Delta_{v, i}-S \cap \partial \Delta_{v, i}+\partial\left(S \cap \Delta_{v, i}\right)
$$

$$
C^{\prime} \cap \Delta_{v, i}-C \cap \Delta_{i}=U_{i}+\partial V_{i}
$$

où $U_{i}=R \cap \Delta_{v, i}-S \cap \partial \Delta_{v, i}+R_{i}$ et $V_{i}=S \cap \Delta_{v, i}+S_{i}$ sont petits,

$$
\sum_{i \in I} M\left(U_{i}\right)+M\left(V_{i}\right)<\text { const. } \epsilon .
$$

Supposons que $i \in I_{1}$. Alors $C^{\prime} \cap \Delta_{v, i}-U_{i}-\partial V_{i}=P_{i}$. Si $t$ est un multiple entier de $k,\left(\pi \circ \delta_{t}\right)_{\sharp}\left(C^{\prime} \cap \Delta_{v, i}-U_{i}\right)$ est un $n$-1-cycle de $T_{\infty}$, homologue à $\left(\pi \circ \delta_{t}\right)_{\sharp} P_{i}$. D'après la définition 8 et le lemme 20 ,

$$
\left|\left[\left(\pi \circ \delta_{t}\right)_{\sharp}\left(C^{\prime} \cap \Delta_{v, i}-U_{i}\right)\right]\right|_{T, \mathbb{R}}=\left|\left[\left(\pi \circ \delta_{t}\right)_{\sharp} P_{i}\right]\right|_{T_{\infty}, \mathbb{R}} .
$$

Le lemme 19 montre que cette norme (une borne inférieure) est atteinte précisément par le cycle $\left(\pi \circ \delta_{t}\right)_{\sharp} P_{i}$. Il vient

$$
\begin{aligned}
M_{t}\left(C^{\prime} \cap \Delta_{v, i}-U_{i}\right)= & t^{1-n} M\left(\left(\delta_{t}\right)_{\sharp}\left(C^{\prime} \cap \Delta_{v, i}-U_{i}\right)\right)=t^{1-n} M\left(\left(\pi \circ \delta_{t}\right)_{\sharp}\left(C^{\prime} \cap \Delta_{v, i}-U_{i}\right)\right) \\
& \geq t^{1-n}\left|\left[\left(\pi \circ \delta_{t}\right)_{\sharp} P_{i}\right]\right|_{T_{\infty}, \mathbb{R}}=M_{\infty}\left(P_{i}\right) .
\end{aligned}
$$


Enfin

$$
\begin{aligned}
M_{\infty}(C)= & \sum_{i \in I_{0}} M_{\infty}\left(C \cap \Delta_{i}\right)+\sum_{i \in I_{1}} M_{\infty}\left(P_{i}\right) \leq \text { const. } \epsilon+\sum_{i \in I_{1}} M_{t}\left(C^{\prime} \cap \Delta_{v, i}-U_{i}\right) \\
& \leq \text { const. } \epsilon+\sum_{i \in I_{1}} M_{t}\left(C^{\prime} \cap \Delta_{v, i}\right)+M_{t}\left(U_{i}\right) \leq M_{t}\left(C^{\prime}\right)+\text { const. } \epsilon .
\end{aligned}
$$

Lemme 25. Soit $C$ un courant entier de dimension $n-1$ dans $R$. Pour tout $\epsilon>0$, il existe un entier $k$ et un courant $(k, \epsilon)$-rationnel $\tilde{C}$ tel que $\|\tilde{C}-C\|_{b}<\epsilon$.

Preuve. On approche d'abord $C$ par une chaîne. Puis on triangule les sous-variétés en présence. Quitte à subdiviser et perturber les simplexes, on peut les supposer affines et en position générale les uns par rapport aux autres. Quitte à subdiviser et perturber, on peut supposer ces simplexes deux à deux disjoints. On est ramené au cas où $C$ est formé d'un seul simplexe affine $\sigma$.

Quitte à translater et perturber, on peut supposer que les sommets $v_{i}$ de $\sigma$ ont des coordonnées rationnelles et $v_{0}=0$. Soit $\ell$ un entier, $P$ le parallélépipède formé sur les vecteurs $\frac{1}{\ell} v_{1}, \ldots, \frac{1}{\ell} v_{n-1}, P_{w}$ le translaté par $w$. Alors $\sigma=\sum_{w} P_{w}+R$ où la somme porte sur les vecteurs $w$ de la forme $w=\sum_{i} \frac{1}{\ell} x_{i} v_{i}$ où les $x_{i}$ sont des entiers naturels tels que $\frac{1}{\ell} \sum_{i}\left(x_{i}+1\right) \leq 1$. Le courant $R$ a son support contenu dans le voisinage de largeur $\frac{1}{\ell}$ de la face opposée à $v_{0}$, et sa masse est de l'ordre de $\frac{1}{\ell}$. On construit facilement une subdivision polyédrale de $R$ qui découpe sur $\sigma$ exactement les parallélépipèdes $P_{w}$. Ceci prouve que $\sigma$ est $(k, \epsilon)$-rationnel pour $\epsilon \sim \frac{1}{\ell}$ et $k$ le plus petit entier tel que les composantes des vecteurs $\frac{k}{\ell} v_{i}$ soient entières.

\subsection{Preuve de la proposition 22}

Soit $t_{j}$ une suite tendant vers $+\infty$ et soit $C_{j}$ une suite de courants entiers fermés sur $R$, qui converge au sens de la topologie b vers $C$. On peut supposer que $M\left(C_{j}\right)$ reste borné.

Fixons $\epsilon>0$. D'après le lemme 25 , il existe un entier $k$ et un courant $(k, \epsilon)$-rationnel $\tilde{C}$ tel que $\|\tilde{C}-C\|_{b}$ $<\epsilon / 3$. Le courant dilaté $\delta\left(C_{j}\right)$ dépend continument de $r$ (en général, $\left\|\delta_{r}(T)-T\right\|_{b} \leq$ const. $(M(T)+M(\partial T)$ ). Il existe donc un $\eta>0$ tel que si $1<r<1+\eta$, alors $\left\|\delta_{r}\left(C_{j}\right)-C_{j}\right\|_{b}<\epsilon / 3$ pour tout $j$. Soit $t>k(\eta+1) / \eta$. Notons $\ell$ la partie entière de $t / k$ et $r=t / k \ell$. Alors $1<r<1+\eta$ et si $j$ est assez grand, $\left\|\delta_{r} C_{j}-\tilde{C}\right\|_{b}<\epsilon$. D'après le lemme 24,

$$
M_{\infty}(\tilde{C}) \leq M_{k \ell}\left(\delta_{r} C_{j}\right)+\text { const. } \epsilon .
$$

Comme $\delta_{t} C_{j}=\delta_{k \ell} \delta_{r} C_{j}$, il vient

$$
M_{\infty}(\tilde{C}) \leq r^{n-1} M_{t}\left(C_{j}\right)+\text { const. } \epsilon .
$$

Pour conclure, on invoque la semi-continuité de la masse (Prop. 21) : lorsque $\epsilon$ tend vers $0, M_{\infty}(\tilde{C})$ est au moins aussi grand que $M_{\infty}(C)$.

\section{Minoration DU PROFIL isopéRIMÉtRIQUe}

\subsection{Réduction aux courants à support dans un compact}

Lemme 26. Soit $R$ un espace vectoriel de dimension $n, E$ un réseau de $R$. On munit $R$ d'une métrique finslérienne E-invariante. Pour tout $\epsilon>0$, il existe une constante const. $(\epsilon)$ telle que pour tout courant entier $C$ de dimension $n$ sur $\mathbb{R}^{n}$, il existe un courant entier $C^{\prime}$ tel que

- $M\left(C^{\prime}\right)=M(C)$;

- $M\left(\partial C^{\prime}\right) \leq M(\partial C)+\epsilon M(C)$;

- $C^{\prime}$ est contenu dans une boule de rayon const. $(\epsilon)$. 
Preuve. On peut supposer que $1 / \epsilon$ est entier. Soit $C$ un courant de dimension $n$ sur $R$. Quitte à remplacer $C$ par sa valeur absolue $|C|$ (voir Rem. 15), on peut supposer que $C$ est positif. Soit $G$ le sous-ensemble de $R$ formé des vecteurs $v$ tels qu'au moins une des composantes de $\epsilon v$ soit entière. Notons $G_{v}$ le translaté de $G$ par $v$. Notons $K$ le cube formé des vecteurs dont toutes les composantes sont comprises entre 0 et $1 / \epsilon$. Alors par Fubini

$$
\int_{K} M\left(C \cap G_{v}\right) d v=n M(C) .
$$

Par conséquent il existe $v \in K$ tel que $M\left(C \cap G_{v}\right) \leq n \epsilon^{n} M(C)$. Pour $w \in E$, notons $C_{w}$ le translaté par $-(1 / \epsilon) w$ de $C \cap(K+(1 / \epsilon) w)$ et $C^{\prime}=\sum_{w} C_{w}$. Comme $C$ est positif, et comme la masse est invariante par translation entière, $M\left(C^{\prime}\right)=M(C)$ et

$$
M\left(\partial C^{\prime}\right) \leq \sum_{w} M\left(\partial C_{w}\right) \leq M(\partial C)+2 M\left(C \cap G_{w}\right) \leq M(\partial C)+2 n \epsilon^{n} M(C)
$$

\subsection{Preuve de l'inégalité $\lim \inf I / I_{\infty} \geq 1$}

Proposition 27. Soit $T$ un tore finslérien muni d'un élément de volume d'intégrale 1 . Soit $T_{\infty}$ le tore finslérien avec élément de volume défini en 8 . Alors les profils isopérimétriques des revêtements universels de $T$ et $T_{\infty}$ satisfont

$$
\lim \inf _{\tau \rightarrow+\infty} I(\tau) / I_{\infty}(\tau) \geq 1
$$

Preuve. Comme en section 6 , on voit le revêtement universel de $T$ comme l'espace vectoriel $R=H_{1}(T, \mathbb{R})$ muni d'une métrique $E$-invariante et d'un élément de volume invariant par translations.

Soit $\tau_{j}$ une suite de réels tendant vers $+\infty$, soient $D_{j}$ des domaines dans $\tilde{T}=R$ de volume $\tau_{j}$ qui réalisent asymptotiquement le minimum du volume du bord, i.e. $\operatorname{Vol}\left(\partial D_{j}\right) /\left(\tau_{j}\right)^{n-1 / n}$ tend vers $\lim \inf I(\tau) / \tau^{n-1 / n}$. Soit $\tilde{D}_{j}$ le domaine $D_{j}$ dilaté de $1 / t_{j}$ où $t_{j}^{n}=\tau_{j}$ de sorte que son volume soit égal à 1 . D'après le corollaire 26 , pour tout $\epsilon>0$, il existe une suite de courants entiers $C_{j}^{\prime}$ contenus dans un compact fixe tels que $M\left(C_{j}^{\prime}\right)=\operatorname{Vol} \tilde{C}_{j}$ et $M_{t_{j}}\left(\partial C_{j}^{\prime}\right) \leq(1+\epsilon) \operatorname{Vol} \partial \tilde{C}_{j}$. D'après le théorème de compacité (Prop. 12), on peut supposer que les suites $C_{j}^{\prime}$ et $\partial C_{j}^{\prime}$ convergent au sens de la topologie $b$ vers un courant entier $C$. En dimension $n$, cela entraîne la convergence des masses, donc $M(C)=1$. Par semi-continuité (Prop. 22),

$$
M_{\infty}(\partial C) \leq \lim \sup M_{t_{j}}\left(\partial C_{j}^{\prime}\right) \leq(1+\epsilon) \lim \inf _{\tau \rightarrow+\infty} I(\tau) / \tau^{n-1 / n}
$$

Ceci est vrai pour tout $\epsilon>0$. D'après la proposition 16, l'inégalité isopérimétrique pour la masse $M_{\infty}$ s'étend aux courants avec la même constante. On conclut que

$$
I_{\infty}(1) \leq M_{\infty}(\partial C) \leq \lim \inf _{\tau \rightarrow+\infty} I(\tau) / \tau^{n-1 / n}
$$

Par homogénéité, $I_{\infty}(\tau) / \tau^{n-1 / n}=I_{\infty}(1)$ donc

$$
\lim \inf _{\tau \rightarrow+\infty} \frac{I(\tau)}{I_{\infty}(\tau)} \geq 1
$$




\section{MAJORATION DU PROFIL ISOPÉRIMÉTRIQUE}

Proposition 28. Soit $T$ un tore finslérien muni d'un élément de volume d'intégrale 1. Soit $T_{\infty}$ le tore finslérien avec élément de volume défini en 8. Alors les profils isopérimétriques des revêtements universels de $T$ et $T_{\infty}$ satisfont

$$
\lim \sup _{\tau \rightarrow+\infty} I(\tau) / I_{\infty}(\tau) \leq 1
$$

Preuve. Soit $T$ un tore finslérien. De nouveau, on voit le revêtement universel $\tilde{T}$ comme une métrique $E=H_{1}(T, \mathbb{Z})$-invariante sur $R=H_{1}(T, \mathbb{R})$ dont l'élément de volume est invariant par translation. On note $|\cdot|_{1}$ la norme quotient de la norme $L^{1}$ sur $R^{*}=H^{1}(T, \mathbb{R})$.

Soit $\epsilon>0$. Soit $\rho$ une norme polyédrale (i.e. dont les boules sont des polyèdres) sur $R$. Il existe un nombre fini de formes linéaires $\beta_{j}$ sur $R$ telles que $\rho=\sup _{j} \beta_{j}$. On interprête les $\beta_{j}$ comme des classes de cohomologie dans $H^{1}(T, \mathbb{R})$. Choisissons des 1-formes différentielles fermées $\alpha_{j} \in \beta_{j}$ telles que $\left\|\alpha_{j}\right\|_{L^{1}(T)}<(1+\epsilon)\left|\beta_{j}\right|_{1}$. Relevons $\alpha_{j}$ au revêtement universel $\tilde{T}=R$ et notons $f_{j}$ sa primitive qui s'annule à l'origine. Posons $\tilde{\rho}=\sup _{j} \beta_{j}$.

Sur le réseau entier, la fonction $f_{j}$ cö̈ncide avec la fonction linéaire $\beta_{j}$. Par conséquent, la différence $\left|\beta_{j}-f_{j}\right|$ est bornée pour chaque $j$, et il en est de même de $|\tilde{\rho}-\rho|$. Notons $V_{j}$ le lieu des points où $\rho=\beta_{j}$. C'est un cône polyédral. Dans $V_{j}$ et en dehors d'un voisinage tubulaire de $\partial V_{j}$ de largeur bornée on a $\tilde{\rho}=f_{j}$.

Soit $\chi_{t, \eta}$ la fonction sur $\mathbb{R}$ qui vaut 1 sur $\left.]-\infty, t\right]$, qui vaut $1-(x-t) / \eta \operatorname{sur}[t, t+\eta]$ et 0 sur $[t+\epsilon,+\infty[$. On va comparer les normes des différentielles des fonctions $u_{t}=\chi_{t, \eta} \circ \rho$ et $\tilde{u}_{t}=\chi_{t, \eta} \circ \tilde{\rho}$.

On choisit un $\eta=\eta(t)$ qui tend vers l'infini avec $t$ mais moins vite que $t$. La fonction $\left|d \tilde{u}_{t}\right|$ est nulle en dehors d'une couronne $\{t-$ const. $\leq \rho \leq t+$ const. $\}$. Elle est bornée par const. $/ \epsilon$ dans la couronne. L'intersection de cette couronne et des voisinages tubulaires des $\partial V_{j}$ est de volume majoré par $t^{n-2}$ donc négligeable. On raisonne donc comme si $\tilde{\rho}$ coïncidait avec $f_{j}$ sur $V_{j}$, et on compare l'intégrale $\int_{V_{j}}\left|d \chi_{t, \eta} \circ f_{j}\right|$ à $\int_{V_{j}}\left|d \chi_{t, \eta} \circ \beta_{j}\right|_{1}$.

Soit $K$ un majorant de $\left|\beta_{j}-f_{j}\right|$. On note $W_{j}$ (resp. $Y_{j}$ ) la réunion des cubes unité à sommets entiers qui rencontrent (resp. sont entièrement contenus dans) la bande $V_{j} \cap\left\{t-K \leq \beta_{j} \leq t+\eta+K\right\}$ (resp. $\left.V_{j} \cap\left\{t+K \leq \beta_{j} \leq t+\eta-K\right\}\right)$. Alors $\left|d \chi_{t, \eta} \circ f_{j}\right|$ est bornée sur $W_{j}$, nulle en dehors de $W_{j}$ et coïncide avec la fonction périodique $\frac{1}{\eta}\left|\alpha_{j}\right|$ sur $Y_{j}$. Comme $\operatorname{Vol}\left(W_{j}\right) / \operatorname{Vol}\left(Y_{j}\right)$ tend vers 1 ,

$$
\int_{V_{j}}\left|d \chi_{t, \eta} \circ f_{j}\right| \sim \int_{Y_{j}}\left|d \chi_{t, \eta} \circ f_{j}\right|=\left\|\alpha_{j}\right\|_{L^{1}(T)} \#\left\{\operatorname{cubes} \subset Y_{j}\right\} \sim \frac{\left\|\alpha_{j}\right\|_{L^{1}(T)}}{\left|\beta_{j}\right|_{1} .} \int_{V_{j}}\left|d \chi_{t, \eta} \circ \beta_{j}\right|_{1} .
$$

Par conséquent $\int\left|d \tilde{u}_{t}\right|<(1+\epsilon) \int\left|d u_{t}\right|_{1}$ pour $t$ assez grand.

On utilise la formule de la coaire (voir Sect. 13)

$$
\int\left|d \tilde{u}_{t}\right|=\int_{0}^{1} \operatorname{Vol}\left\{\tilde{u}_{t}=s\right\} d s
$$

pour choisir une valeur de $s \in] 0,1\left[\right.$ telle que $\operatorname{Vol}\left\{\tilde{u}_{t}=s\right\} \leq \int\left|d \tilde{u}_{t}\right|$. La formule de la coaire donne à nouveau

$$
\int\left|d u_{t}\right|_{1}=\frac{1}{\eta} \int_{\{t \leq \rho \leq t+\eta\}}|d \rho|_{1}=\frac{1}{\eta} \int_{t}^{t+\eta} M_{\infty}(\{\rho=s\}) d s \sim M_{\infty}(\{\rho=t\}) .
$$

Comparons le volume du domaine $D=\left\{\tilde{u}_{t} \leq s\right\}$ à $\tau=\operatorname{Vol}\{\rho \leq t\}$. Si $K$ est un majorant pour $|\tilde{\rho}-\rho|$, alors $D$ contient $\{\rho \leq t-K\}$ et est contenu dans $\{\rho \leq t+\eta+K\}$ donc $(1-K / t)^{n} \tau \leq \operatorname{Vol}(D) \leq(1+\eta / t+K / t)^{n} \tau$. En particulier, pour $t$ assez grand, $(1-\epsilon) \tau \leq \operatorname{Vol}(D) \leq(1+\epsilon) \tau$.

D'après le théorème de Brunn (Prop. 30 et son cas d'égalité), les domaines optimaux pour l'inégalité isopérimétrique relative à la norme $|\cdot|_{1}$ (sur le dual) sont convexes et symétriques. On peut donc les approcher par des polyèdres convexes et symétriques. On conclut que pour tout $\epsilon>0$, pour $\tau$ assez grand, il existe un $\tau^{\prime}$ tel que $\left|\tau^{\prime}-\tau\right|<\epsilon \tau$ et $I\left(\tau^{\prime}\right)<(1+\epsilon) I_{\infty}\left(\tau^{\prime}\right)$. 
Pour conclure, on remarque que la fonction $I$ est sous-additive : $I(a+b) \leq I(a)+I(b)$. En effet, deux domaines de volumes $a$ et $b$ peuvent être rendus disjoints en translatant l'un d'eux d'un vecteur entier. Cela fournit un domaine de volume $a+b$ et les volumes des bords s'ajoutent. D'autre part, par comparaison avec une métrique euclidienne, $I(\tau) \leq$ const. $\tau^{n-1 / n}$ et $I_{\infty}(\tau) \geq$ const. $\tau^{n-1 / n}$. Par conséquent,

$$
I(\tau) \leq I\left(\tau^{\prime}\right)+I\left(\tau-\tau^{\prime}\right) \leq(1+\epsilon) I_{\infty}\left(\tau^{\prime}\right)+\text { const. }(\epsilon \tau)^{n-1 / n} \leq\left(1+\text { const. } \epsilon^{n-1 / n}\right) I_{\infty}(\tau) .
$$

\section{Preuve Du THÉORÈme 1}

Il reste seulement à passer du cas particulier où le tore a un volume égal à 1 au cas général.

Lemme 29. Soit $V$ une variété finslérienne de dimension $n$ munie d'un élément de volume $\omega$. Notons $|\cdot|_{\lambda, \infty}$ la norme quotient de la norme $L^{\infty}$ sur $H^{n-1}(V, \mathbb{R})$ correspondant au choix de l'élément de volume $\lambda \omega$. Alors

$$
|\cdot|_{\lambda, \infty}=\lambda^{-1}|\cdot|_{1, \infty} .
$$

Notons $I_{\lambda}$ le profil isopérimétrique de la variété $V$ munie de la même métrique finslérienne mais de l'élément de volume $\lambda \omega$. Alors

$$
I_{\lambda}(\tau)=\lambda I_{1}\left(\lambda^{-1} \tau\right) .
$$

Notons $I^{\mu}$ le profil isopérimétrique de la variété $V$ munie du même élément de volume $\omega$ mais d'une métrique finslérienne multipliée par $\mu$ (de sorte que les distances sont multipliées par $\mu$ ). Alors

$$
I^{\mu}(\tau)=\mu^{-1} I_{1}(\tau) .
$$

Preuve. L'intégrand de Poincaré $|\cdot|_{\lambda}$ sur $\Lambda^{n-1} T^{*} V$ est

$$
|\phi|_{\lambda}=\sup \{\alpha \wedge \phi / \lambda \omega ;|\alpha| \leq 1\}=\lambda^{-1}|\phi|_{1} .
$$

Par conséquent, sur $H^{n-1}$,

$$
|\cdot|_{\lambda, \infty}=\lambda^{-1}|\cdot|_{1, \infty}
$$

Notons $\operatorname{Vol}_{\lambda}$ les volumes en dimension $n$ et $M_{\lambda}$ les volumes en dimension $n-1$ pour le choix d'élément de volume $\lambda \omega$. Clairement, $\operatorname{Vol}_{\lambda}=\lambda \operatorname{Vol}_{1}$. Si $C$ est un courant de dimension $n-1$,

$$
M_{\lambda}(C)=\sup \left\{C(\phi) /|\phi|_{\lambda} ; \phi n-1-\text { forme }\right\}=\lambda M_{1}(C) .
$$

Soit $D$ un domaine tel que $\operatorname{Vol}_{\lambda}(D)=\tau$ et $M_{\lambda}(\partial D)=I_{\lambda}(\tau)$. Alors

$$
I_{\lambda}(\tau)=\lambda M_{1}(\partial D)=\lambda I_{1}\left(\operatorname{Vol}_{1}(D)\right)=\lambda I_{1}(\tau / \lambda) .
$$

Notons $\mathrm{Vol}^{\mu}$ les volumes en dimension $n$ et $M^{\mu}$ les volumes en dimension $n-1$ pour le choix d'élément de volume $\omega$ et la métrique multipliée par $\mu$. La norme sur les 1-formes est divisée par $\mu$. Par conséquent l'intégrand de Poincaré est

Si $C$ est un courant de dimension $n-1$,

$$
|\phi|^{\mu}=\sup \left\{\alpha \wedge \phi /|\alpha|^{\mu} \omega ; \alpha 1-\text { forme }\right\}=\mu|\phi|^{1} .
$$

$$
M^{\mu}(C)=\sup \left\{C(\phi) /|\phi|^{\mu} ; \phi n-1-\text { forme }\right\}=\mu^{-1} M^{1}(C) .
$$

Soit $D$ un domaine tel que $\operatorname{Vol}^{\mu}(D)=\tau$ et $M^{\mu}(\partial D)=I^{\mu}(\tau)$. Alors

$$
I^{\mu}(\tau)=\mu^{-1} M^{1}(\partial D)=\mu^{-1} I_{1}\left(\operatorname{Vol}^{1}(D)\right)=\mu^{-1} I_{1}(\tau) .
$$




\subsection{Preuve de la proposition 9}

Soit $\lambda=\int_{T} \omega$ de sorte que pour $\omega_{1}=\lambda^{-1} \omega, \int_{T} \omega_{1}=1$. Notons $I_{1}$ le profil isopérimétrique du revêtement universel de $T$ muni de l'élément de volume $\omega_{1}$. Notons $I_{1, \infty}$ le profil isopérimétrique asymptotique correspondant (c'est celui de l'espace vectoriel $R=H^{n-1}(T, \mathbb{R})$ muni de la norme $|\cdot|_{1, \infty}$ où $|\cdot|_{1, \infty}=\lambda|\cdot|_{\infty}$. D'après les propositions 27 et 28 ,

$$
\lim _{\tau \rightarrow+\infty} I_{1}(\tau) / I_{1, \infty}(\tau)=1 .
$$

D'après le lemme 29, $I(\tau)=\lambda I_{1}\left(\lambda^{-1} \tau\right)$, et, posant $\mu=\lambda^{-1}, I_{\infty}(\tau)=\lambda I_{1, \infty}(\tau)$. D'autre part, $I_{1, \infty}(\tau)$ $=\lambda^{n-1 / n} I_{1, \infty}\left(\lambda^{-1} \tau\right)$, donc

$$
\lim _{\tau \rightarrow+\infty} I(\tau) / I_{\infty}(\tau)=\lambda^{1-n / n}=\lambda=\left(\int_{T} \omega\right)^{1-n / n}
$$

\subsection{Inégalité isopérimétrique pour les espaces vectoriels normés}

Elle est connue depuis 1887. Pour une preuve moderne de l'inégalité de Brunn, voir l'appendice de Gromov [25], p. 126.

Proposition 30. (Brunn [7]). Soit $R$ un espace vectoriel normé de dimension $n$ muni d'un élément de volume tel que la boule unité soit de volume 1 . On munit $\Lambda^{n-1} R^{*}$ de l'intégrand de Poincaré. Le profil isopérimétrique de $R$ est alors $I(\tau)=n \tau^{n-1 / n}$.

Preuve. Dans [25], on trouve la version équivalente (d'après la Prop. 16)

$$
n\|u\|_{L^{n / n-1}} \leq\||d u|\|_{1} .
$$

Remarque. Cas d'égalité dans l'inégalité de Brunn.

Le théorème suivant est dû à Taylor [32]. On suppose $\mathbb{R}^{n}$ muni d'un intégrand convexe (i.e. d'une norme sur les $n$-1-formes). Soit $C$ un courant entier de dimension $n$. Si $M(\partial T)=n M(T)^{n-1 / n}$, alors $C$ est le courant d'intégration sur un convexe homothétique à la forme de Wulff définie par l'intégrand (i.e. une boule de la norme Poincaré duale).

\subsection{Preuve du théorème 1}

Il reste à passer du cas où la boule unité est de volume 1 au cas général. On utilise l'isomorphisme 17. Soit $R=H^{n-1}(T, \mathbb{R})$ muni de la norme $|\cdot|_{\infty}$, soit $E=H^{n-1}(T, \mathbb{Z})$ et soit $\omega_{\infty}$ l'élément de volume d'intégrale 1 sur $R / E$. Soit $B$ la boule unité de $R$. On pose $\lambda=\int_{B} \omega_{\infty}$ et $\omega_{1}=\lambda^{-1} \omega_{\infty}$ de sorte que $\int_{B} \omega_{1}=1$. D'après la proposition 30 , le profil relatif à $\omega_{1}$ vaut $I_{1}(\tau)=n \tau^{n-1 / n}$. Le lemme 29 donne

$$
I_{\infty}(\tau)=\lambda I_{1}\left(\lambda^{-1} \tau\right)=n\left(\int_{B} \omega_{\infty}\right)^{1 / n} \tau^{n-1 / n}
$$

Enfin, lorsque $r$ est grand, le volume pour $\omega_{\infty}$ de la boule de rayon $r$ est équivalent au nombre de points entiers dans cette boule, donc $\int_{B} \omega_{\infty}$ est égal au volume asymptotique. 


\subsection{Convergence des domaines extrémaux}

La preuve donne la convergence en topologie $b$ des domaines qui réalisent le minimum du volume du bord à volume intérieur fixé.

Proposition 31. Soit $T$ un tore riemannien. Identifions son revêtement universel à l'espace vectoriel $R=H_{1}(T, \mathbb{R})$ muni d'une métrique riemannienne périodique et dont l'élément de volume est constant. Notons I son profil isopérimétrique. Pour tout $\epsilon>0$ il existe $\tau_{\epsilon}$ et $\eta_{\epsilon}$ tels que si $D \subset R$ est un domaine de volume $\geq \tau_{\epsilon}$ et tel que

$$
\operatorname{Vol}(\partial D) \leq\left(1+\eta_{\epsilon}\right) c_{\infty} \operatorname{Vol}(D)^{n-1 / n}
$$

alors il existe une boule $B$ pour la distance associée à la norme $|\cdot|_{1}^{*}$ duale de la norme $L^{1}$ sur $R^{*}=H^{1}(T, \mathbb{R})$ telle que

$$
\|D-B\|_{b}<\epsilon \operatorname{Vol}(D)
$$

Preuve. Par l'absurde. Etant donné un domaine $D$ de $R$, le normaliser consiste à le dilater jusqu'à ce que son volume soit égal à 1 et le translater pour amener son centre de gravité à l'origine. Si la proposition 31 est fausse, il existe une suite $D_{j}$ de domaines presque extrémaux qui une fois normalisés ne convergent pas vers $B$. Il ne coûte rien de supposer que $\operatorname{Vol} T=1$. On a montré en 7 qu'une sous-suite des $\tilde{D}_{j}$ converge vers un courant $D$ de masse 1 et tel que

$$
M_{\infty}(\partial D) \leq \liminf \operatorname{Vol}\left(D_{j}\right)^{1-n / n} M\left(\partial D_{j}\right)=c_{\infty} .
$$

Comme le profil isopérimétrique de la masse $M_{\infty}$ est exactement $c_{\infty} \tau^{n-1 / n}$ (Prop. 9), $D$ réalise le cas d'égalité dans l'inégalité de Brunn (Prop. 30). Par conséquent, $D$ est une boule de la norme sur $R$. Comme les $\tilde{D}_{j}$ ont leur centre de gravité à l'origine, il en est de même de $D$. Par conséquent, $D=B$, contradiction.

Remarque. Sur les courants de dimension maximum, la norme b ne dépend pas de la métrique de référence, seulement de l'élément de volume.

\subsection{Preuve du théorème 2}

Si $\phi$ est une forme différentielle de degré $n-1$ sur $T$, alors par l'inégalité de Hölder

$$
\|\phi\|_{L^{n / n-1}} \leq \operatorname{Vol}(T)^{n-1 / n}\|\phi\|_{L^{\infty}} .
$$

Par conséquent, dans le groupe $H^{n-1}(T, \mathbb{Z})$, la boule de rayon $r$ pour la norme $|\cdot|_{\infty}$ est contenue dans la boule de rayon $r \operatorname{Vol}(T)^{n-1 / n}$ pour la norme $|\cdot|_{n / n-1}$. Il vient

$$
V A\left(H^{n-1}(T, \mathbb{Z}),|\cdot|_{\infty}\right) \leq \operatorname{Vol}(T)^{n-1} V A\left(H^{n-1}(T, \mathbb{Z}),|\cdot|_{n / n-1}\right)
$$

donc avec le théorème 1

$$
c_{\infty} \leq n\left(V A\left(H^{n-1}(T, \mathbb{Z}),|\cdot|_{n / n-1}\right)\right)^{1 / n} .
$$

Montrons que pour une métrique riemannienne plate sur $T$, on a l'égalité

$$
c_{\text {eucl }}=n\left(V A\left(H^{n-1}(T, \mathbb{Z}),|\cdot|_{n / n-1}\right)\right)^{1 / n} .
$$

On peut supposer que $\operatorname{Vol}(T)=1$. Alors $T=R / E$ où $R$ est un espace euclidien et $E \subset R$ un réseau de volume 1. Les formes différentielles à coefficients constants minimisent toutes les normes $L^{p}$ dans leur classe de cohomologie. En effet, la norme $L^{p}$ est strictement convexe, atteint son minimum, et une forme différentielle $\phi$ à coefficients constants est solution de l'équation d'Euler-Lagrange $d *\left(|\phi|^{p-2} \phi\right)=0$ qui caractérise le minimum. Par conséquent, l'isomorphisme $R^{*} \rightarrow H^{1}(T, \mathbb{R})$ qui envoie un covecteur sur la classe de la 1-forme différentielle invariante par translation sur $T$ est une isométrie. Cet isomorphisme envoie $E^{*}$ sur $H^{1}(T, \mathbb{Z})$. Or le réseau $E^{*}$ 
est aussi de volume 1. Par conséquent, $V A\left(H^{1}(T, \mathbb{Z}),|\cdot|_{2}\right)=V A\left(E^{*}\right)=\beta_{n}$ est le volume de la boule unité de $R$. Par conséquent $n\left(V A\left(H^{n-1}(T, \mathbb{Z}),|\cdot|_{n / n-1}\right)\right)^{1 / n}=n \beta_{n}^{1 / n}=c_{\text {eucl }}$.

En dimension 2, toute classe conforme contient une métrique plate, donc $V A\left(H^{1}(T, \mathbb{Z}),|\cdot|_{2}\right)=\pi$ pour tout 2-tore riemannien.

L'inégalité de Hölder

entraîne

$$
|\cdot|_{1} \leq \operatorname{Vol}(T)^{1 / n}|\cdot|_{n / n-1}
$$

$$
V A\left(H^{n-1}(T, \mathbb{Z}),|\cdot|_{n / n-1}\right) \leq \operatorname{Vol}(T) V A\left(H^{n-1}(T, \mathbb{Z}),|\cdot|_{1}\right) .
$$

Avec la proposition 17, il vient

$$
\operatorname{Vol}(T) V A\left(H^{n-1}(T, \mathbb{Z}),|\cdot|_{1}\right)=\operatorname{Vol}(T) V A\left(H_{1}(T, \mathbb{Z}),|\cdot|_{\mathbb{R}}\right)=V A(\tilde{T}) .
$$

Avec le théorème 1, on trouve

$$
c_{\infty} \leq n V A\left(H^{n-1}(T, \mathbb{Z}),|\cdot|_{n / n-1}\right)^{1 / n} \leq n V A(\tilde{T})^{1 / n} .
$$

Remarque. En dimension 2, l'inégalité $\pi=V A\left(H^{1}(T, \mathbb{Z}),|\cdot|_{2}\right) \leq V A(\tilde{T})$ est due à Babenko [3].

En toutes dimensions, l'inégalité $c_{\infty} \leq n V A(\tilde{T})^{1 / n}$ s'obtient aussi directement en appliquant l'inégalité isopérimétrique aux boules du revêtement universel.

\section{Preuve DU ThÉORÈme 3}

\subsection{Volume asymptotique de la norme duale}

Lemme 32. Soit $E$ un groupe abélien libre de rang $n$, muni d'une norme $N$. Soit $E^{*}=H o m(E, \mathbb{Z})$ muni de la norme duale $N^{*}$. Notons $\beta_{n}$ le volume de la boule unité euclidienne. Alors

$$
V A(E, N) V A\left(E^{*}, N^{*}\right) \leq \beta_{n}^{2}
$$

avec égalité si et seulement si la norme $N$ est euclidienne.

Preuve. Soit $R=E \otimes \mathbb{R}$ et soit $B$ la boule unité de $(R, N)$. Choisissons une structure euclidienne auxiliaire sur $R$ telle que le réseau $E$ soit de volume 1, de sorte que $V A(E, N)=\operatorname{Vol} B$. Ramenons $E^{*}$ et $N^{*}$ dans $R$ au moyen de la structure euclidienne. Soit $B^{*}$ la boule unité de $N^{*}$. Alors $E^{*}$ est de volume 1 et $V A\left(E^{*}, N^{*}\right)=\operatorname{Vol} B^{*}$. Enfin $B^{*}$ est le polaire du convexe $B$. L'inégalité de Blaschke-Santalo (voir [4,29] et [18], p. 165) énonce que $\mathrm{Vol} B \mathrm{Vol} B^{*} \leq \beta_{n}^{2}$ avec égalité si et seulement si $B$ est un ellipsoïde.

\subsection{Preuve du théorème 3}

Soit $T$ un tore riemannien de dimension $n$. Le théorème de Burago et Ivanov [5], s'énonce

$$
V A(\tilde{T}) \geq \beta_{n}
$$

avec égalité si et seulement si $T$ est plat. Or

$$
V A(\tilde{T})=\operatorname{Vol}(T) V A\left(H_{1}(T, \mathbb{Z}),|\cdot| \mathbb{R}\right) .
$$

D'après la proposition 17 , les normes $|\cdot|_{\mathbb{R}}$ sur $H_{1}(T, \mathbb{Z})$ et $|\cdot|_{\infty}$ sur $H^{1}(T, \mathbb{Z})$ sont duales, donc avec le lemme 32,

$$
V A\left(H_{1}(T, \mathbb{Z}),|\cdot|_{\mathbb{R}}\right) V A\left(H^{1}(T, \mathbb{Z}),|\cdot|_{\infty}\right) \leq \beta_{n}^{2} .
$$


Ces deux inégalités donnent

$$
V A\left(H^{1}(T, \mathbb{Z}),|\cdot|_{\infty}\right) \leq \beta_{n} \operatorname{Vol}(T)
$$

d'où

$$
c_{\infty} \leq n \beta_{n}^{1 / n}\left(\frac{V A\left(H^{n-1}(T, \mathbb{Z}),|\cdot|_{\infty}\right)}{V A\left(H^{1}(T, \mathbb{Z}),|\cdot|_{\infty}\right)^{n-1}}\right)^{1 / n} .
$$

S'il y a égalité (par exemple si $n=2$ et $c_{\infty}=2 \sqrt{\pi}$ ), on doit avoir l'égalité dans le théorème de Burago-Ivanov, donc la métrique est plate.

\section{MÉtriques DE GRANDE CONSTANTE ISOPÉRIMÉtRIQUE ASYMPtotique}

Proposition 33. En toute dimension, il existe des tores riemanniens de dimension $n$ dont la constante isopérimétrique asymptotique $c_{\infty}$ est arbitrairement petite.

Si $n \geq 3$, il existe des tores riemanniens de dimension $n$ dont la constante isopérimétrique asymptotique $c_{\infty}$ est arbitrairement grande.

Preuve. Petite constante. Par un changement conforme de métrique, on augmente le volume dans un petit voisinage $B$ d'un point. Cela affecte peu la masse en dimension $n-1$, car faire éviter $B$ à une hypersurface coûte peu d'aire.

On part d'une métrique plate sur $T^{n}$. Soit $B$ une boule de rayon $\epsilon$. Comme un sous-tore affine passe dans ce voisinage un nombre de fois qui est proportionnel à son volume, il faut pour lui faire contourner la boule $B$ multiplier son volume par $\left(1+\right.$ const. $\left.\epsilon^{n-1}\right)$. Ainsi, même si par un changement conforme de métrique on augmente dramatiquement le volume de $B$, la norme $|\cdot|_{\mathbb{R}}$ sur $H_{n-1}(T, \mathbb{Z})$ augmente au plus d'un facteur $\left(1+\right.$ const. $\left.\epsilon^{n-1}\right)$. D'après la proposition 17 , la norme $|\cdot|_{\infty}$ sur $H^{n-1}(T, \mathbb{Z})$ diminue peu, donc le volume asymptotique de $H^{n-1}(T, \mathbb{Z})$ augmente peu, et par le théorème 1 , la constante $c_{\infty}$ diminue beaucoup.

Grande constante. On part de la métrique produit sur $T=(\mathbb{R} / \mathbb{Z})^{n}$. On la perturbe en gardant le même volume mais en augmentant dramatiquement la norme $|\cdot|_{\mathbb{R}}$ sur $H_{n-1}(T, \mathbb{Z})$. Alors, d'après le théorème 1 , la constante $c_{\infty}$ augmente beaucoup. Dans un premier temps, on modifie la métrique au voisinage d'une géodésique $\gamma\left(\right.$ le premier facteur du produit $\left.(\mathbb{R} / \mathbb{Z})^{n}\right)$, en la rendant très grande dans les directions orthogonales à $\gamma$, et très petite dans la direction de $\gamma$, de sorte que l'élément de volume ne change pas. Cela suffit à augmenter la masse de la classe duale de $[\gamma]$. En dimension $>2$, on peut répéter cette chirurgie dans des tubes disjoints, chacun parallèle à l'un des facteurs. Cela suffit pour augmenter toutes les masses en dimension $n-1$.

Écrivons $T=\mathbb{R} / \mathbb{Z} \times T^{\prime}$ avec la métrique initiale $g=d t^{2}+g^{\prime}$. Notons $r$ la distance à l'origine de $T^{\prime}$. Fixons $\epsilon>0$. Notons $U_{1}$ le voisinage tubulaire de largeur $\epsilon$ du premier facteur. Choisissons deux fonctions lisses $a_{\epsilon}$ et $b_{\epsilon} \operatorname{sur} \mathbb{R}$ qui valent 1 en dehors de l'intervalle $]-\epsilon, \epsilon\left[\right.$ et telles que $a_{\epsilon}=\epsilon^{n-1}$ (resp. $\left.b_{\epsilon}=1 / \epsilon^{2}\right)$ sur $]-\epsilon / 2, \epsilon / 2[$. Posons

$$
g_{\epsilon}=a_{\epsilon}^{2} d t^{2}+b_{\epsilon}^{2}(r) g^{\prime} \quad \text { dans } \quad U_{1}, \quad g_{\epsilon}=g \quad \text { ailleurs. }
$$

Le volume de $g_{\epsilon}$ reste voisin de 1 . Soit $e \in H_{n-1}(T, \mathbb{Z})$ une classe dont le nombre d'intersection avec la classe d'homologie du premier facteur est $\lambda \neq 0$. Soit $W \in e$ un représentant de $e$. Alors la restriction à $W \cap U_{1}$ de la projection sur $\left(T^{\prime}, b_{\epsilon}^{2}(r) g^{\prime}\right)$ est de degré $\lambda$. Comme elle dimininue le volume, on a

$$
\operatorname{Vol}\left(W \cap U_{1}\right) \geq \text { const. }|\lambda| \epsilon^{1-n} .
$$

Comme la dimension $n$ est au moins 3 , on peut placer $n$ tubes $U_{i}$ parallèles chacun à un facteur et deux à deux disjoints, et greffer dans chacun une métrique isométrique à $g_{\epsilon}$. Si $e \in H_{n-1}(T, \mathbb{Z})$ a pour composantes $\left(\lambda_{1}, \ldots, \lambda_{n}\right)$ dans la base naturelle, alors

$$
|e|_{\mathbb{R}} \geq \text { const. } \epsilon^{1-n}\left(\sum_{i=1}^{n}\left|\lambda_{i}\right|\right) .
$$


On en tire par dualité pour la classe de la $n-1$-forme $\phi=\sum \lambda_{i} d t_{1} \wedge \cdots \wedge d x_{i-1} \wedge d x_{i+1} \wedge \cdots \wedge d x_{n}$

$$
|[\phi]|_{\infty} \leq \text { const. } \epsilon^{n-1}\left(\max \left|\lambda_{i}\right|\right)
$$

donc

$$
V A\left(H^{n-1}(T, \mathbb{Z}),|\cdot|_{\infty}\right) \geq \text { const. } \epsilon^{1-n} .
$$

\section{Solides COVALENTS}

Dans cette section, on construit une suite de problèmes isopérimétriques continus (des métriques riemanniennes périodiques) qui constituent une approximation du problème isopérimétrique discret étudié dans le théorème 4. La donnée est un ensemble discret d'atomes et de liaisons (i.e. de segments disjoints reliant deux atomes et affectés d'une énergie positive) dans $\mathbb{R}^{3}$.

On construit des métriques riemanniennes ayant les propriétés suivantes.

- Elles donnent un très grand volume à un petit voisinage de chaque atome. En dehors de ces voisinages, le volume est faible.

- Elles donnent à chaque surface une aire au moins proportionnelle à la somme des énergie des liaisons qu'elle coupe. En dehors de voisinages des liaisons, l'aire est faible.

S'il est aisé d'approcher chaque ensemble fini d'atomes par un "domaine tubulaire" dont le volume est proportionnel au nombre d'atomes dans $B$ et l'aire du bord proportionnelle à l'énergie de surface de $B$, la construction réciproque est délicate. En effet, lorsqu'un atome $a$ est proche du bord du domaine $D$, faut-il ou non le prendre? Si le domaine $D$ est presque optimal pour le problème isopérimétrique continu, on peut montrer qu'après nettoyage il contient franchement tout atome $a$ (i.e. il contient ou bien presque tout le volume du voisinage de $a$, ou bien une très faible proportion de ce volume). De même, son bord coupe franchement les liaisons entre atomes. Mais cela nécessite un contrôle des propriétés isopérimétriques de la métrique construite dans les régions où elle varie beaucoup, et rend la preuve particulièrement laborieuse.

\subsection{Comparaison des problèmes isopérimétriques discret et continu}

On appelera solide covalent la donné d'un ensemble $A$ uniformément discret et uniformément dense dans $\mathbb{R}^{n}$, et d'une fonction énergie de liaison $e$ positive et symétrique sur $A \times A$ à support dans un voisinage borné de la diagonale.

Étant donnée une partie finie $B$ de $A$, on note

$$
E(\partial B)=\sum_{a_{1} \in B, a_{2} \notin B} e\left(a_{1}, e_{2}\right) .
$$

On note $I_{d}$ la fonction sur les entiers positifs qui donne pour chaque $N$ le minimum de $E(\partial B)$ sur toutes les parties $B$ de $A$ à $N$ éléments. De principes généraux (voir [17] Chap. 6), il résulte que la fonction $I_{d}$ est encadrée par deux fonctions de la forme const. $\tau^{n-1 / n}$.

Définition 34. Soit $g$ une métrique riemanienne sur $\mathbb{R}^{n}$ et $(A, e)$ un solide covalent de $\mathbb{R}^{n}$. On dit qu'une partie finie $B$ de $A$ et un domaine $D$ de $\mathbb{R}^{n}$ sont $v_{\lambda}, s_{\lambda}, \epsilon$-comparables si

$$
(1-\epsilon) v_{\lambda} \# B \leq \operatorname{Vol}_{g}(D) \leq(1+\epsilon) v_{\lambda} \# B
$$

et

$$
(1-\epsilon) s_{\lambda} E(\partial B) \leq \operatorname{Aire}_{g}(\partial D) \leq(1+\epsilon) s_{\lambda} E(\partial B) .
$$


Proposition 35. Il existe une famille de métriques riemanniennes lisses $g_{\lambda}$ (de profil isopérimétrique $I_{\lambda}$ ) sur $\mathbb{R}^{3}$ et des nombres $\epsilon_{\lambda}$ et $\epsilon_{\lambda}^{\prime}$ tendant vers 0 lorsque $\lambda$ tend vers $+\infty$ tels que toute partie finie $B$ de $A$ soit $v_{\lambda}, s_{\lambda}, \epsilon_{\lambda}$-comparable à un domaine $D$ de $\mathbb{R}^{3}$ contenu dans un voisinage de largeur bornée (pour la métrique euclidienne) de B. Inversement, tout domaine $D$ de $\mathbb{R}^{3}$ de volume suffisamment grand et presque optimal, i.e. tel que Aire ${ }_{\lambda}(\partial D) \leq\left(1+\epsilon_{\lambda}^{\prime}\right) I_{\lambda}\left(\operatorname{Vol}_{\lambda}(D)\right)$ est $v_{\lambda}, s_{\lambda}, \epsilon_{\lambda}$-comparable à une partie finie $B$ de $A$.

Si l'ensemble $A$ et la fonction e sont périodiques, il en est de même des métriques $g_{\lambda}$.

La preuve de la proposition 35 occupe les sections 12.2 à 12.8 .

\subsection{Construction des métriques $g_{\lambda}$}

Dans $\mathbb{R}^{3}$, nous allons utiliser simultanément deux métriques riemanniennes, la métrique euclidienne $g$ de référence, et la métrique $g_{\lambda}$. Celle-ci n'interviendra qu'à travers son élément de volume $\mathrm{Vol}_{\lambda}$ et l'élément d'aire Aire $_{\lambda}$ qu'elle induit sur les surfaces. La distance associée à $g_{\lambda}$ ne jouera aucun rôle. Ainsi une boule $B(a, r)$ sera toujours relative à la métrique euclidienne.

Dans $\mathbb{R}^{3}$, on trace les segments de droite d'extrémités $a_{1}$ et $a_{2} \in A$ tels que $e\left(a_{1}, a_{2}\right)>0$. On les perturbe un peu pour obtenir des arcs d'intérieurs deux à deux disjoints. On modifie la métrique euclidienne seulement au voisinage de ces arcs.

Fixons des réels positifs $r$ et $r^{\prime}$ tels que les boules de rayon $r$ centrées aux points de $A$ soient deux à deux disjointes et tels que les voisinages tubulaires de largeur $r^{\prime}$ des arcs soient deux à deux disjoints hors des boules de rayon $r$ centrées sur $A$. L'existence de ces constantes résulte des hypothèses uniformes sur l'ensemble $A$. En effet, soit $R>0$ tel que $e\left(a_{1}, a_{2}\right)>0$ si $\left|a_{2}-a_{1}\right|>R$. Comme $A$ est uniformément discret, le nombre de points de $A$ dans toute boule de rayon $R$ est borné uniformément, donc le nombre de segments tracés dans une boule de rayon $R$ est borné, et on peut les disjoindre uniformément.

On choisit des fonctions $b_{\lambda}$ sur $\mathbb{R}_{+}$telles que $b_{\lambda}$ est constante sur $\left[0, r^{\prime}\right]$, égale à 1 en dehors d'un intervalle $\left[0, r^{\prime \prime}\left[\right.\right.$ un tout petit peu plus grand. Plus précisément, on note $s_{\lambda}=\int_{0}^{r^{\prime \prime}} 2 \pi b_{\lambda}(\rho) \rho d \rho$ et on s'arrange pour que

$$
s_{\lambda}^{-1} \int_{0}^{r^{\prime \prime}} 2 \pi b_{\lambda}(\rho) \rho d \rho
$$

tende vers 1 quand $\lambda$ tend vers $+\infty$.

Dans le voisinage tubulaire de largeur $r^{\prime \prime}$ de l'arc reliant $a_{1}$ à $a_{2}$, en coordonnées normales à l'arc, la métrique $g_{\lambda}$ satisfait $g_{\lambda} \geq d z^{2}+e\left(a_{1}, a_{2}\right) b_{\lambda}(\rho)\left(d \rho^{2}+\rho^{2} d \theta^{2}\right)$ avec égalité en dehors des boules de rayon $r$ centrées aux extrémités.

Soit $a$ un point de $A$ d'où partent plusieurs arcs. On ajoute toutes les métriques $d z^{2}+e\left(a_{1}, a_{2}\right) b_{\lambda}(\rho)\left(d \rho^{2}\right.$ $\left.+\rho^{2} d \theta^{2}\right)$ correspondant à tous ces arcs. Notons $v_{\lambda}^{\prime}$ le volume de la boule $B(a, r)$ pour la métrique obtenue. Soit $\tilde{r}$ un réel compris entre $r^{\prime \prime}$ et $r$, suffisamment proche de $r$ pour que $\operatorname{Vol}(B(a, r) \backslash B(a, \tilde{r}))<\frac{1}{2} \operatorname{Vol}(B(a, r))$. On augmente encore la métrique dans un tout petit voisinage de $B(a, \tilde{r})$ de sorte que sur $B(a, \tilde{r})$ elle soit proportionnelle à la métrique euclidienne. On note $v_{\lambda}=\operatorname{Vol}_{\lambda} B(a, r)$ le volume de la boule de rayon $r$ pour la métrique obtenue. On s'arrange pour que $s_{\lambda}$ tende vers $+\infty$ et que $s_{\lambda}^{6} / v_{\lambda}$ et $v_{\lambda}^{\prime} / v_{\lambda}$ tendent vers 0 .

En dehors des arcs, la métrique reste essentiellement égale à la métrique euclidienne. Pour les arcs qui sont des segments de droites, la condition $b=1$ au bord suffit pour le raccordement. Au bord du voisinage tubulaire d'un arc non rectiligne, il y a une modification mineure à faire.

L'élément de volume $d \mathrm{Vol}_{\lambda}$ (resp. l'élément d'aire $d \mathrm{Aire}_{\lambda}$ induit sur les surfaces) de la métrique $g_{\lambda}$ est partout plus grand que celui $d \mathrm{Vol}$ (resp. $d$ Aire) de la métrique riemannienne de référence. On a partout les inégalités

$$
d \mathrm{Vol}_{\lambda} \leq \tilde{v}_{\lambda} d \mathrm{Vol}, \quad \text { et } \quad d \mathrm{Aire}_{\lambda} \leq \tilde{v}_{\lambda}^{2 / 3} d \mathrm{Aire}
$$


où $\tilde{v}_{\lambda} / v_{\lambda}$ tend vers 1 lorsque $\lambda$ tend vers $+\infty$. En dehors de la réunion des boules de rayon $r$ centrées aux points de $A$, on peut améliorer ces inégalités en

$$
d \mathrm{Vol}_{\lambda} \leq \tilde{s}_{\lambda} d \mathrm{Vol}, \quad \text { et } d \mathrm{Aire}_{\lambda} \leq \tilde{s}_{\lambda} d \text { Aire },
$$

où $\tilde{s}_{\lambda} / s_{\lambda}$ tend vers 1 lorsque $\lambda$ tend vers $+\infty$.

\subsection{Propriétés isopérimétriques locales des métriques $g_{\lambda}$}

On utilise le résultat classique suivant, voir par exemple [17] chapitre 6.

Lemme 36. Soit $\Omega$ un domaine contenu dans une boule $\beta$ de l'espace euclidien $\mathbb{R}^{n}$, et dont le volume est inférieur à celui de la boule. Notons $S=\partial \Omega \backslash \partial \beta$ la partie du bord de $\Omega$ qui est à l'intérieur de la boule. On a l'inégalité

$$
\operatorname{Vol}(\Omega) \leq \text { const. Aire }(S)^{n / n-1} \text {. }
$$

Soit $S_{\lambda}$ la borne supérieure des aires des bords des boules $B(a, r)\left(S_{\lambda}\right.$ est du même ordre de grandeur que $\left.s_{\lambda}\right)$.

Lemme 37. Soit $a \in A$. Soit $S$ une surface qui partage la boule $B(a, r)$ en deux domaines $\Omega$ et $\Omega^{\prime}$ tels que $\operatorname{Vol}_{\lambda}(\Omega) \leq \operatorname{Vol}_{\lambda}\left(\Omega^{\prime}\right)$. Si $\operatorname{Aire}_{\lambda}(S) \leq S_{\lambda}^{2}$ alors

$$
\operatorname{Vol}_{\lambda}(\Omega) \leq \epsilon_{\lambda} v_{\lambda}
$$

où $\epsilon_{\lambda}$ tend vers 0 lorsque $\lambda$ tend vers $+\infty$. De plus

$$
\operatorname{Vol}(\Omega) \leq \frac{1}{2} \operatorname{Vol} B(a, r)
$$

Preuve. Comme $B(a, \tilde{r})$ munie de la métrique $g_{\lambda}$ est isométrique à une boule euclidienne, on peut appliquer le lemme 36 à $\tilde{S}=S \cap B(a, \tilde{r})$ et $\tilde{\Omega}=\Omega \cap B(a, \tilde{r})$ (ou a son complémentaire $\tilde{\Omega}^{\prime}$ ). Il vient

$$
\min \left\{\operatorname{Vol}_{\lambda}(\tilde{\Omega}), \operatorname{Vol}_{\lambda}\left(\tilde{\Omega}^{\prime}\right)\right\} \leq \text { const. } \operatorname{Aire}_{\lambda}(\tilde{S})^{3 / 2}
$$

Le plus petit des volumes de $\tilde{\Omega}$ et de son complémentaire est très petit devant $v_{\lambda}$, donc le plus grand est de l'ordre de $v_{\lambda}$, bien supérieur au volume de $\Omega$ (au plus la moitié de $v_{\lambda}$ ), donc c'est bien $\tilde{\Omega}$ et non son complémentaire qui a le plus petit volume. Il vient

$$
\operatorname{Vol}_{\lambda}(\Omega) \leq \operatorname{Vol}_{\lambda}\left(\Omega^{\prime}\right)+\operatorname{Vol}_{\lambda}(B(a, r) \backslash B(a, \tilde{r})) \leq \text { const. } s_{\lambda}^{3}+v_{\lambda}^{\prime}
$$

qui est petit devant $v_{\lambda}$. Enfin

$$
\operatorname{Vol} \tilde{\Omega} \sim v_{\lambda}^{-1} \operatorname{Vol}_{\lambda} \tilde{\Omega}
$$

est petit. Comme $\operatorname{Vol}(B(a, r) \backslash B(a, \tilde{r}))<\frac{1}{2} \operatorname{Vol}(B(a, r))$,

$$
\operatorname{Vol} \Omega \leq \operatorname{Vol} \tilde{\Omega}+\operatorname{Vol}(B(a, r) \backslash B(a, \tilde{r})) \leq \frac{1}{2} \operatorname{Vol}(B(a, r))
$$

lorsque $\lambda$ est grand.

Lemme 38. Soit $a \in A$ et soit $\Omega$ une partie de la boule $B(a, r)$ dont le complémentaire a un volume (pour $\left.g_{\lambda}\right)$ petit devant $v_{\lambda}$. On fixe un arc $\left[a, a_{2}\right]$ issu de $a$, un plan $P$ perpendiculaire à cet arc, muni de la métrique induite par $g_{\lambda}$. A chaque point de ce plan correspond un segment parallèle à l'arc (i.e. paramétré en coordonnées normales par $t$ à $r$ et $\theta$ constants). Soit $Z$ l'ensemble des segments qui ne rencontrent pas $\Omega$. Alors l'aire de $Z$ est petite devant $s_{\lambda}$. 
Preuve. Notons $\beta$ l'intersection de la boule $B(a, \tilde{r})$ et du voisinage tubulaire de l'axe. Notons $\pi: \beta \rightarrow P$ la projection le long des segments parallèles à l'arc. Notons $\beta^{\prime}$ la boule de rayon $r^{\prime}$ dans $P$, et $\gamma=\pi^{-1}\left(\beta^{\prime}\right)$. Munie de la métrique $g_{\lambda}, B(a, \tilde{r})$ est isométrique à une boule euclidienne dont le volume est de l'ordre de $v_{\lambda}$. L'ensemble $\gamma$ est isométrique à l'intersection de cette boule avec un cylindre, et son volume est du même ordre de grandeur. Munie de la métrique induite par $g_{\lambda}, \beta^{\prime}$ est isométrique à une boule euclidienne dont le volume est de l'ordre de $s_{\lambda}$, et la projection $\pi: \gamma \rightarrow \beta^{\prime}$ est linéaire et surjective. Son jacobien $J(\pi)$ est constant et la longueur des fibres de l'ordre de $v_{\lambda}^{1 / 3}$. D'après la formule de la coaire, si $Z \subset \beta^{\prime}$,

$$
\operatorname{Vol}_{\lambda}\left(\pi^{-1} Z\right)=\int_{Z}\left(\int_{\pi^{-1}(z)} J(\pi)^{-1}\right) d z \sim J(\pi)^{-1} v_{\lambda}^{1 / 3} \operatorname{Aire}_{\lambda}(Z) .
$$

En faisant $Z=\beta^{\prime}$, on trouve $J(\pi) \sim s_{\lambda} / v_{\lambda}^{2 / 3}$. En appliquant cette formule à l'ensemble $Z$ de l'énoncé, pour lequel $\pi^{-1} Z$ est contenu dans le complémentaire de $\Omega$, on trouve que $s_{\lambda}^{-1} \operatorname{Aire}_{\lambda}(Z) \leq v_{\lambda}^{-1} \operatorname{Vol}_{\lambda}(B(x, r) \backslash \Omega)$ est petit.

Lemme 39. Soit $f$ une fonction sur $\mathbb{R}^{3}$. Soient $a_{1}, a_{2}$ deux points de $A$ tels que e $\left(a_{1}, a_{2}\right)>0$, donc reliés par un tube T. On note $\tilde{f}\left(a_{i}\right)$ la médiane de $f$ sur la boule $B\left(a_{i}, r\right)$ pour la mesure $\operatorname{Vol}_{\lambda}$, i.e.

$$
\operatorname{Vol}_{\lambda}\left(B\left(a_{i}, r\right) \cap\left\{f \leq \tilde{f}\left(a_{i}\right)\right\}\right) \leq \frac{1}{2} v_{\lambda} \leq \operatorname{Vol}_{\lambda}\left(B\left(a_{i}, r\right) \cap\left\{f \geq \tilde{f}\left(a_{i}\right)\right\}\right) .
$$

Soit $C>0$. On suppose que pour $i=1,2$,

$$
\int_{B\left(a_{i}, r\right)}|d f|_{\lambda} d \mathrm{Vol}_{\lambda} \leq C s_{\lambda}
$$

Alors

$$
\int_{T}|d f|_{\lambda} d \operatorname{Vol}_{\lambda} \geq\left(1-\epsilon_{\lambda}\right) s_{\lambda}\left(\left|\tilde{f}\left(a_{2}\right)-\tilde{f}\left(a_{1}\right)\right|-\epsilon_{\lambda} C\right)
$$

où $\epsilon_{\lambda}$ tend vers 0 quand $\lambda$ tend vers $+\infty$.

Preuve. Posons $\phi_{\lambda}=C / s_{\lambda}$. D'après la formule de la coaire,

$$
\int_{\tilde{f}\left(a_{i}\right)-\phi_{\lambda}}^{\tilde{f}\left(a_{i}\right)} \operatorname{Aire}_{\lambda}\left(B\left(a_{i}, r\right) \cap\{f=s\}\right) d s \leq \phi_{\lambda}^{-1} \int_{B\left(a_{i}, r\right)}|d f|_{\lambda} d \operatorname{Vol}_{\lambda} .
$$

Par conséquent, il existe $s \in] \tilde{f}\left(a_{i}\right)-\phi_{\lambda}, \tilde{f}\left(a_{i}\right)[$ tel que

$$
\operatorname{Aire}_{\lambda}\left(B\left(a_{i}, r\right) \cap\{f=s\}\right) \leq \phi_{\lambda}^{-1} \int_{B\left(a_{i}, r\right)}|d f|_{\lambda} d \mathrm{Vol}_{\lambda} .
$$

Par hypothèse, cette intégrale est majorée par $C s_{\lambda}$, donc $\operatorname{Aire}_{\lambda}\left(B\left(a_{i}, r\right) \cap\{f=s\}\right) \leq s_{\lambda}^{2} \leq S_{\lambda}^{2}$ pour $\lambda$ grand. D'après le lemme $37, \operatorname{Vol}_{\lambda}\left(B\left(a_{i}, r\right) \cap\{f \leq s\}\right)$ est petit devant $v_{\lambda}$. On peut donc appliquer le lemme 38 . Dans le tube $T$, utilisons les coordonnées $(t, \rho, \theta)$ normales à l'arc reliant les deux atomes $a_{1}$ et $a_{2}$. L'ensemble $Z$ des segments $\sigma_{\rho, \theta}$ tels que $\sigma_{\rho, \theta} \cap B(a, r)$ rencontre $\{f \leq s\}$ est d'Aire $\lambda_{\lambda}$ petite devant $s_{\lambda}$. On fait le même travail dans l'intervalle $] \tilde{f}\left(a_{i}\right), \tilde{f}\left(a_{i}\right)+\phi_{\lambda}\left[\right.$. On trouve un ensemble $Z^{\prime}$ d'Aire $\lambda_{\lambda}$ petite devant $s_{\lambda}$ telle que pour $(\rho, \theta) \notin Z^{\prime}$, sur le segment $\sigma_{\rho, \theta}$ et dans chacune des boules $B\left(a_{i}, r\right)$, la fonction $f$ est à valeurs dans $] \tilde{f}\left(a_{i}\right)-\phi_{\lambda}, \tilde{f}\left(a_{i}\right)+\phi_{\lambda}[$. On note $\sigma_{\rho, \theta}^{\prime}$ la partie du segment $\sigma_{\rho, \theta}$ située en dehors des deux boules $B\left(a_{i}, r\right)$ et $e_{i}(\rho, \theta)$ ses extrémités. Hors des boules $B\left(a_{i}, r\right)$, la métrique $g_{\lambda}$ est un produit $d t^{2}+e\left(a_{1}, a_{2}\right) b_{\lambda}(\rho)\left(d \rho^{2}+\rho^{2} d \theta^{2}\right)$. Par conséquent

$$
|d f|_{\lambda} \geq\left|\frac{\partial f}{\partial t}\right|
$$


donc

$$
\int_{\sigma_{\rho, \theta}^{\prime}}|d f|_{\lambda} d t \geq\left|\int_{\sigma_{\rho, \theta}^{\prime}} d f\right|=\left|f\left(e_{2}(\rho, \theta)\right)-f\left(e_{1}(\rho, \theta)\right)\right| .
$$

Si $(\rho, \theta) \notin Z^{\prime},\left|f\left(e_{i}(\rho, \theta)\right)-\tilde{f}\left(a_{i}\right)\right| \leq \phi_{\lambda}$, donc

$$
\int_{T}|d f|_{\lambda} d \operatorname{Vol}_{\lambda} \geq\left(s_{\lambda}-\operatorname{Aire}_{\lambda}\left(Z^{\prime}\right)\right) e\left(a_{1}, a_{2}\right)\left(\left|\tilde{f}\left(a_{2}\right)-\tilde{f}\left(a_{1}\right)\right|-2 \phi_{\lambda}\right) .
$$

\subsection{Domaine tubulaire associé à un ensemble d'atomes}

Soit $B$ une partie finie de l'ensemble $A$ des atomes. On lui associe un domaine $D$ de $\mathbb{R}^{3}$ comme suit. On prend la réunion des boules de rayon $r$ centrées sur $B$, et on y ajoute des tubes : un voisinage tubulaire de l'arc reliant $a_{1}$ à $a_{2}$ pour chaque paire telle que $a_{1} \in B$ et $a_{2} \notin B$. Par construction, $D \cap A=B$. On a les inégalités

$$
v_{\lambda} \# B \leq \operatorname{Vol}_{\lambda}(D) \leq v_{\lambda} \# B+\text { const. } s_{\lambda} \# B
$$

alors que le volume de son bord satisfait

$$
s_{\lambda} E(\partial B) \leq \operatorname{Aire}_{\lambda}(\partial D) \leq s_{\lambda} E(\partial B)+\text { const. } \# B
$$

où les constantes ne dépendent ni de $\lambda$ ni de $B$. En effet, les contributions importantes au volume de $D$ viennent des boules centrées sur $B$. Comme le nombre de tubes issus de chaque sommet est borné, les tubes sont en nombre proportionnel à $\# B$. Les contributions importantes à l'aire du bord viennent des sphères de rayon $r$ centrées en des points de $B$ qui coupent des liaisons entre points de $B$ et points du complémentaire. L'aire du bord de chaque tube est bornée et leur nombre est proportionnel à \#B.

On peut en particulier majorer le profil isopérimétrique $I_{\lambda}$ de $g_{\lambda}$. En effet, si $D$ est le domaine construit à partir d'un ensemble fini $B$, l'inégalité $I_{\lambda}\left(\operatorname{Vol}_{\lambda}(D)\right) \leq \operatorname{Aire}_{\lambda}(\partial D)$ se traduit par

$$
I_{\lambda}(\tau) \leq \text { const. } \frac{s_{\lambda}}{v_{\lambda}^{2 / 3}} \tau^{2 / 3}
$$

pour $\tau$ multiple entier de $v_{\lambda}$. D'après le théorème 1 , cette inégalité reste vraie pour tout $\tau$ assez grand.

\subsection{Ensemble d'atomes associé à un domaine presque optimal}

Inversement, étant donné un domaine $D$ de $\mathbb{R}^{3}$, on va lui associer un sous-ensemble fini $B$ de $A$ tel que $v_{\lambda} \# B$ soit voisin de $\operatorname{Vol}_{\lambda} D$.

On se livre à un nettoyage préliminaire : on modifie $D$ de sorte que le bord $\partial D$ ne laisse que peu d'aire dans chaque boule de rayon $r$ centrée en un point de $A$. Soit $B_{0}$ l'ensemble des points $a$ de $A$ tels que Aire $_{\lambda}(\partial D \cap B(a, r))>S_{\lambda}$. Pour chaque $a \in B_{0}$, si on ajoute ou retire à $D$ la boule $B(a, r)$, l'Aire $\lambda_{\lambda}$ de $\partial D$ diminue. Notons $\tau$ la somme des volumes des intersections $D \cap B(a, r)$, lorsque $a$ décrit $B_{0}$. Soit $k$ la partie entière de $\tau / v_{\lambda}$. Si on ajoute à $D k$ des boules centrées sur des points de $B_{0}$ et on retranche à $D$ les $\# B_{0}-k$ autres, on obtient un nouveau domaine $D^{\prime}$ tel que

$$
\left|\operatorname{Vol}_{\lambda} D^{\prime}-\operatorname{Vol}_{\lambda} D\right| \leq v_{\lambda} \quad \text { et } \quad \operatorname{Aire}_{\lambda}\left(D^{\prime}\right) \leq \operatorname{Aire}_{\lambda}(D) .
$$

Par construction, pour tout $a \in A, \operatorname{Aire}_{\lambda}\left(\partial D^{\prime} \cap B(a, r)\right) \leq S_{\lambda}$. 
Soit $B$ l'ensemble des atomes $a \in A$ tels que $\operatorname{Vol}_{\lambda}\left(D^{\prime} \cap B(a, r)\right) \geq \frac{1}{2} v_{\lambda}$. Comme $S_{\lambda} \leq S_{\lambda}^{2}$, on peut appliquer le lemme 37 à l'intersection $D^{\prime} \cap B(a, r)$ pour tout $a \in B$. On a $\operatorname{Vol}_{\lambda}\left(D^{\prime} \cap B(a, r)\right) \geq\left(1-\epsilon_{\lambda}\right) v_{\lambda}$. Par conséquent

$$
v_{\lambda} \# B \leq \operatorname{Vol}_{\lambda}\left(D^{\prime}\right) /\left(1-\epsilon_{\lambda}\right) .
$$

Autrement dit, lorsque $\lambda$ est grand, le nombre d'atomes dans $B$ est majoré par la valeur souhaitée.

\subsection{Minoration du nombre d'atomes}

Montrons que si $D$ est de volume suffisamment grand et est compétitif du point de vue isopérimétrique, i.e. si $\operatorname{Aire}_{\lambda}(\partial D) \leq$ const. $I_{\lambda}\left(\operatorname{Vol}_{\lambda}(D)\right)$, alors $v_{\lambda} \# B \geq \operatorname{Vol}_{\lambda}\left(D^{\prime}\right)\left(1-\epsilon_{\lambda}\right)$. Notons

$$
D^{\prime}=D_{1} \cup D_{2} \cup \bigcup_{j} \Omega_{j}
$$

où $D_{2}$ est l'intersection de $D^{\prime}$ avec la réunion des boules de rayon $r$ centrées aux points de $B$ et les $\Omega_{j}$ sont les intersections de $D^{\prime}$ avec les boules de rayon $r$ centrées aux points $a_{j}$ de $A \backslash B$. Notons $S_{j}=\partial D^{\prime} \cup B\left(a_{j}, r\right)$ la partie du bord de $\Omega_{j}$ qui n'est pas dans le bord de $B\left(a_{j}, r\right)$. Grâce au nettoyage préliminaire,

$$
\operatorname{Aire}_{\lambda}\left(S_{j}\right) \leq S_{\lambda}
$$

pour tout $j$. Par construction de l'ensemble $B, \operatorname{Vol}_{\lambda} \Omega_{j} \leq \frac{1}{2} v_{\lambda}$ donc d'après le lemme 37 , Vol $\Omega_{j} \leq \frac{1}{2} \operatorname{Vol} B\left(a_{j}, r\right)$. D'après le lemme 36 ,

$$
\operatorname{Vol}\left(\Omega_{j}\right) \leq \text { const. Aire }\left(S_{j}\right)^{3 / 2} \leq \text { const. Aire }{ }_{\lambda}\left(S_{j}\right)^{3 / 2}
$$

car Aire $\leq$ Aire $_{\lambda}$. Il vient

$$
\sum_{j} \operatorname{Vol}\left(\Omega_{j}\right) \leq \text { const. } \sum_{j} \operatorname{Aire}_{\lambda}\left(S_{j}\right)^{3 / 2} \leq \text { const. } S_{\lambda}^{1 / 2} \sum_{j} \operatorname{Aire}_{\lambda}\left(S_{j}\right) \leq \text { const. } S_{\lambda}^{1 / 2} \operatorname{Aire}_{\lambda}\left(\partial D^{\prime}\right) .
$$

Avec l'hypothèse que $\operatorname{Aire}_{\lambda}(\partial D) \leq$ const. $I_{\lambda}\left(\operatorname{Vol}_{\lambda}(D)\right)$ et l'estimation obtenue plus haut du profil $I_{\lambda}$, il vient

$$
\begin{aligned}
\operatorname{Vol}_{\lambda}\left(D^{\prime}\right) & \leq s_{\lambda} \operatorname{Vol}\left(D_{1}\right)+\operatorname{Vol}_{\lambda}\left(D_{2}\right)+v_{\lambda} \sum_{j} \operatorname{Vol}\left(\Omega_{j}\right) \leq s_{\lambda} \operatorname{Vol}_{\lambda}\left(D^{\prime}\right)+v_{\lambda} \# B+\text { const. } S_{\lambda}^{1 / 2} \operatorname{Aire}_{\lambda}\left(\partial D^{\prime}\right) \\
& \leq v_{\lambda} \# B+\text { const. }\left(s_{\lambda} \operatorname{Vol}_{\lambda}\left(D^{\prime}\right)+s_{\lambda}^{1 / 2} \frac{s_{\lambda}}{v_{\lambda}^{2 / 3}} \operatorname{Vol}_{\lambda}\left(D^{\prime}\right)^{2 / 3}\right)
\end{aligned}
$$

Ceci prouve que lorsque $\operatorname{Vol}_{\lambda}\left(D^{\prime}\right)$ est assez grand, $\operatorname{Vol}_{\lambda}\left(D^{\prime}\right) \leq\left(1+\epsilon_{\lambda}\right) v_{\lambda} \# B$.

\subsection{Minoration de l'aire du bord}

Comparons maintenant $\operatorname{Aire}_{\lambda}\left(\partial D^{\prime}\right)$ à $E(\partial B)$. Soient $a_{1}$ et $a_{2}$ des points de $A$ tels que $a_{1} \in B, a_{2} \notin B$ et $e\left(a_{1}, a_{2}\right)>0$. Notons $T$ le tube reliant $a_{1}$ à $a_{2}$. Montrons que

$$
s_{\lambda} e\left(a_{1}, a_{2}\right) \leq\left(1+\epsilon_{\lambda}\right) \operatorname{Aire}_{\lambda}\left(T \cap \partial D^{\prime}\right) .
$$

Notons $\Omega=B\left(a_{1}, r\right) \cap D^{\prime}$ et $\Omega^{\prime}=B\left(a_{2}, r\right) \backslash D^{\prime}$. Le lemme 37 montre que le complémentaire de $\Omega$ (resp. de $\left.\Omega^{\prime}\right)$ a un volume petit devant $v_{\lambda}$. Notons $X$ la boule de rayon $r^{\prime \prime}$ dans un plan perpendiculaire à l'arc $\left[a_{1}, a_{2}\right]$. Son aire vaut $e\left(a_{1}, a_{2}\right) s_{\lambda}$. D'après le lemme 38, l'ensemble $Z \subset X$ des segments parallèles à l'arc $\left[a_{1}, a_{2}\right]$ dont l'intersection avec $B\left(a_{1}, r\right)$ est entièrement contenue dans $\Omega$ ou bien dont l'intersection avec $B\left(a_{2}, r\right)$ est 
entièrement contenue dans $\Omega^{\prime}$ ) est d'aire petite devant $s_{\lambda}$. Tout segment qui n'est pas dans $Z$ rencontre donc à la fois $D^{\prime}$ et son complémentaire. Il coupe donc le bord $\partial D^{\prime}$. Ceci prouve que

$$
\operatorname{Aire}_{\lambda}\left(T \cap \partial D^{\prime}\right) \geq \operatorname{Aire}_{\lambda}(X \backslash Z) \geq s_{\lambda} e\left(a_{1}, a_{2}\right)\left(1-\epsilon_{\lambda}\right)
$$

où $\epsilon_{\lambda}$ tend vers 0 .

En sommant sur les liaisons $\left[a_{1}, a_{2}\right]$ entre points de $B$ et points du complémentaire, on conclut que

$$
\operatorname{Aire}_{\lambda}\left(\partial D^{\prime}\right) \geq s_{\lambda} E(\partial B)\left(1-\epsilon_{\lambda}\right)
$$

\subsection{Majoration de l'aire du bord}

Montrons que si le domaine $D$ est presque optimal, i.e. si $\operatorname{Aire}_{\lambda}(\partial D) \leq\left(1+\epsilon_{\lambda}\right) I_{\lambda}\left(\operatorname{Vol}_{\lambda}(D)\right)$, alors

$$
\operatorname{Aire}_{\lambda}(\partial D) \leq\left(1+\epsilon_{\lambda}\right) E(\partial B)
$$

En effet, notons $\Delta$ le domaine tubulaire associé à l'ensemble $B$. Il satisfait

$$
\operatorname{Vol}_{\lambda}(\Delta) \sim v_{\lambda} \# B \sim \operatorname{Vol}_{\lambda}(D)
$$

et

$$
\operatorname{Aire}_{\lambda}(\partial \Delta) \sim s_{\lambda} E(\partial B)
$$

Par définition du profil isopérimétrique,

$$
I_{\lambda}\left(\operatorname{Vol}_{\lambda} \Delta\right) \leq \operatorname{Aire}_{\lambda}(\partial \Delta)
$$

or si $\operatorname{Vol}_{\lambda}(D)$ est suffisamment grand, la fonction $I_{\lambda}$ est presque une fonction puissance donc

$$
\operatorname{Aire}_{\lambda}(\partial D) \leq\left(1+\epsilon_{\lambda}\right) I_{\lambda}\left(\operatorname{Vol}_{\lambda}(D)\right) \sim I_{\lambda}\left(\operatorname{Vol}_{\lambda} \Delta\right) \leq \operatorname{Aire}_{\lambda}(\partial \Delta) \sim s_{\lambda} E(\partial B)
$$

Ceci achève la preuve de la proposition 35.

\section{Preuve DU ThÉORÈme 4}

\subsection{Normes induites sur $H_{1}$ par les métriques $g_{\lambda}$}

Lemme 40. Soit $(A, e)$ un solide covalent invariant par un groupe $G$ de translations. La métrique $g_{\lambda}$ induit une métrique riemannienne, encore notée $g_{\lambda}$, sur le tore $T=\mathbb{R}^{3} / G$. On note $|\cdot|_{1, \lambda}$ la norme sur $H_{1}(T, \mathbb{R})$ quotient de la norme $L^{1}$ sur les 1 -formes différentielles calculée au moyen de la métrique $g_{\lambda}$. On note $|\cdot|_{1, \lambda}^{*}$ la norme duale sur $H_{1}(T, \mathbb{R})$. Lorsque $\lambda$ tend vers $+\infty$, la boule unité $\beta_{\lambda}$ de la norme $\left.s_{\lambda}|\cdot|\right|_{1, \lambda} ^{*}$ converge vers la boule unité $\beta$ de la norme cristalline pour la distance de Hausdorff.

Preuve. Notons $\tilde{\Gamma}$ le graphe dont l'ensemble des sommets est $A$ et dont les arêtes correspondent aux couples $\left(a_{1}, a_{2}\right)$ tels que $e\left(a_{1}, a_{2}\right)>0$. Soit $\Gamma=\tilde{\Gamma} / G$. C'est un graphe fini qu'on peut imaginer plongé dans le tore $T$, en réalisant ses arêtes par les arcs qui interviennent dans la construction des métriques $g_{\lambda}$. On note $\Delta$ le quotient par $G$ du domaine tubulaire associé à $A$ entier. Soit $\ell$ une forme linéaire sur $\mathbb{R}^{3}$ et $f$ un cocycle représentant $\ell$, au sens de la définition 4. La fonction $g=f-\ell$ sur $A$ est $G$-invariante. Elle est donc définie sur les sommets de $\Gamma$. Prolongeons là à $\Gamma$ de sorte qu'elle soit constante dans le $r$-voisinage des sommets, et affine sur le reste des arcs. Prolongeons à nouveau cette fonction au domaine tubulaire $\Delta$ via la projection orthogonale sur les arcs. Prolongeons là enfin au reste du tore $T$ au moyen d'une troncature. En lui ajoutant la fonction linéaire $\ell$, on trouve un prolongement $f^{\prime}$ de $f$ à $\mathbb{R}^{3}$. Alors $\alpha_{f}=d f^{\prime}$ passe au quotient en une 1-forme fermée sur le tore $T$, 
identiquement nulle sur les boules $B(a, r)$ centrées sur les points de $A / G$, et telle que sur les tubes reliant deux points de $A / G$, les composantes tangentielles de $\alpha_{f}$ sont nulles. Clairement, la norme de $\alpha_{f}$ pour la métrique $g_{\lambda}$ est indépendante de $\lambda$ (au facteur multiplicatif $s_{\lambda}$ près). Elle satisfait

$$
\left\|\alpha_{f}\right\|_{1, \lambda} \leq s_{\lambda}\|f\|_{1}+\text { const. }|\ell|_{\text {ref }}
$$

où $|\cdot|_{\text {ref }}$ désigne la norme euclidienne de référence sur le dual de $\mathbb{R}^{3}$. Cela signifie que

$$
|\ell|_{1, \lambda} \leq s_{\lambda}|\ell|_{\text {cris }}^{*}+\text { const. }|\ell|_{\text {ref }}
$$

Inversement, soit $\alpha$ une 1-forme fermée sur $T$ dont la classe de cohomologie est celle de la forme linéaire $\ell$. On va minorer sa norme $L^{1}$ par une quantité voisine de $s_{\lambda}|\ell|_{\text {cris }}$. On peut donc supposer que cette norme $L^{1}$ est inférieure à $s_{\lambda}$ const. $|\ell|_{r e f}$. Sur le revêtement universel $\mathbb{R}^{3}, \alpha$ est exacte, $\alpha=d f$. Si $a \in A$, on définit $\tilde{f}_{\lambda}(a)$ comme la valeur médiane de $f$ sur la boule $B(a, r)$ pour la mesure $\operatorname{Vol}_{\lambda}$, i.e.

$$
\operatorname{Vol}_{\lambda}\left(B(a, r) \cap\left\{f \leq \tilde{f}_{\lambda}(a)\right\}\right) \leq \frac{1}{2} v_{\lambda} \leq \operatorname{Vol}_{\lambda}\left(B(a, r) \cap\left\{f \geq \tilde{f}_{\lambda}(a)\right\}\right)
$$

Alors $\tilde{f}_{\lambda}$ est un cocycle représentant $\ell$. Lorsque $\lambda$ tend vers $+\infty, \tilde{f}_{\lambda}$ converge vers la restriction de $f$ à $A$. Le lemme 39 montre que la norme $L^{1}$ de la 1-forme $\alpha$ pour la métrique $g_{\lambda}$ satisfait

$$
\|\alpha\|_{L^{g_{\lambda}}} \geq\left(1-\epsilon_{\lambda}\right) s_{\lambda}\left(\left\|\tilde{f}_{\lambda}\right\|_{1}-\epsilon_{\lambda} \text { const. }|\ell|_{r e f}\right)
$$

où $\epsilon_{\lambda}$ tend vers 0 quand $\lambda$ tend vers $+\infty$. Par conséquent

$$
|\ell|_{1, \lambda} \geq\left(1-\epsilon_{\lambda}\right) s_{\lambda}\left(|\ell|_{\text {cris }}^{*}-\epsilon_{\lambda}|\ell|_{\text {ref }}\right) .
$$

On conclut que si $|\cdot|_{\text {cris }}^{*}$ est une norme, alors $|\cdot|_{1, \lambda}$ est équivalente à $s_{\lambda}|\ell|_{\text {cris }}^{*}$ lorsque $\lambda$ tend vers $+\infty$, uniformément sur le dual de $\mathbb{R}^{3}$. Par conséquent les boules unité des normes duales convergent au sens de Hausdorff.

Le lemme 41 montre que cette conclusion persiste même si $|\cdot|_{c r i s}^{*}$ n'est pas une norme.

Lemme 41. Soit $(V,|\cdot|)$ un espace vectoriel normé de dimension finie. Soit $\epsilon>0$ et soient $|\cdot|_{0}^{*}$ et $|\cdot|{ }_{1}^{*}$ deux semi-normes sur le dual $V^{*}$ telles que

$$
|\cdot|_{0}^{*}-\epsilon|\cdot|^{*} \leq|\cdot|_{1}^{*} \leq|\cdot|_{0}^{*}+\epsilon|\cdot|^{*} .
$$

Alors la distance de Hausdorff entre les boules unité

$$
\beta_{i}=\left\{v \in V ; \forall v \in V, \ell(v) \leq|\ell|_{i}^{*}\right\}
$$

est inférieure à const. $\epsilon$.

Preuve. Notons $V_{1}^{*} \subset V^{*}$ le sous-espace vectoriel des formes linéaires de norme $|\ell|_{0}^{*}$ nulle, et $V_{0} \subset V$ le sous-espace des vecteurs annulés par $V_{1}^{*}$. Soit $V_{1} \subset V$ un supplémentaire de $V_{0}$ et $V_{0}^{*} \subset V^{*}$ son annulateur. Comme $|\cdot|_{0}^{*}$ est une norme sur $V_{0}^{*}$, si $\ell=\ell_{0}+\ell_{1} \in V_{0}^{*}+V_{1}^{*}=V^{*}$, alors

$$
|\ell|_{1}^{*} \leq(1+\text { const. } \epsilon)\left|\ell_{0}\right|_{0}^{*}+\epsilon\left|\ell_{1}\right|^{*} .
$$

Par dualité entre norme $L^{1}$ et norme $L^{\infty}$, si $v=v_{0}+v_{1} \in V_{0}+V_{1}=V$,

$$
|v|_{1} \geq \max \left\{\frac{1}{1+\text { const. } \epsilon}\left|v_{0}\right|_{0}, \frac{1}{\epsilon}\left|v_{1}\right|\right\} .
$$


Par conséquent, si $v=v_{0}+v_{0} \in \beta_{1}$, alors $\left|v_{0}\right|_{0} \leq 1+$ const. $\epsilon$ et $\left|v_{1}\right| \leq \epsilon$. En particulier $v^{\prime}=\frac{1}{1+\text { const. } \epsilon} v_{0} \in \beta_{0}$ et $\left|v-v^{\prime}\right| \leq$ const. $\epsilon$. Ceci prouve que la boule $\beta_{1}$ est contenue dans le const. $\epsilon$-voisinage de $\beta_{0}$. En échangeant les rôles de $\beta_{1}$ et de $\beta_{0}$, on montre que $\beta_{0}$ est contenue dans le const. $\epsilon$-voisinage de $\beta_{1}$. On conclut que la distance de Hausdorff entre $\beta_{1}$ et $\beta_{0}$ est au plus égale à const. $\epsilon$.

\subsection{Preuve du théorème 4}

On raisonne par l'absurde. Soit $N_{j}$ une suite d'entiers tendant vers $+\infty$. Soit $B_{j}$ une partie à $N_{j}$ éléments de $A$ qui est approximativement optimale, i.e. telle que $E\left(\partial B_{j}\right) \leq\left(1+\epsilon_{j}\right) I_{d}\left(N_{j}\right)$ où la suite $\epsilon_{j}$ tend vers 0 , mais qui ne converge pas au sens de la distance $\delta$ introduite en 5 vers une boule de la norme cristalline. Notons $D_{j}$ le domaine tubulaire construit à partir de $B_{j}$.

La proposition 34 montre qu'il existe $j_{\lambda}$ tel que pour $j \geq j_{\lambda}, D_{j}$ soit approximativement optimal pour toutes les métriques $g_{\lambda}$, précisément,

$$
j \geq j_{\lambda} \Rightarrow \operatorname{Aire}_{\lambda}\left(\partial D_{j}\right) \leq\left(1+\epsilon_{j, \lambda}\right) I_{\lambda}\left(\operatorname{Vol}_{\lambda}\left(D_{j}\right)\right)
$$

où $\epsilon_{j, \lambda}$ tend vers 0 quand $j$ et $\lambda$ tendent vers $+\infty$ avec $j \geq j_{\lambda}$.

Par hypothèse, le solide $(A, e)$ est périodique, de réseau de Bravais $G$. D'après [22], il existe pour chaque $\lambda$ un difféomorphisme $\psi_{\lambda}$ du tore $\mathbb{R}^{3} / G$, isotope à l'identité et tel que l'élément de volume de la métrique $\psi_{\lambda}^{*} g_{\lambda}$ soit constant. On note $\tilde{\psi}_{\lambda}$ un relèvement de $\psi_{\lambda}$ au revêtement universel. C'est un difféomorphisme de $\mathbb{R}^{3}$ qui déplace les points d'une distance bornée, disons, par $\delta_{\lambda}$.

D'après la proposition 31, pour chaque $\lambda$ et lorsque $j$ est assez grand, le courant $I_{D_{j}}$ est proche en norme $b$ d'une boule $\beta_{j, \lambda}$ de la norme $|\cdot|_{1, \lambda}^{*}$ induite sur $H_{1}\left(\mathbb{R}^{3} / G, \mathbb{R}\right)=\mathbb{R}^{3}$ par la métrique $g_{\lambda}$, précisément

$$
\epsilon_{j, \lambda}=\left\|I_{\tilde{\psi}_{\lambda}\left(D_{j}\right)}-I_{\beta_{j, \lambda}}\right\|_{b} / \operatorname{Vol}\left(D_{j}\right)
$$

tend vers 0 lorsque, à $\lambda$ fixé, $j$ tend vers l'infini. En dimension maximum, la norme $b$ coïncide avec la masse, i.e. avec le volume. Par conséquent le nombre $\epsilon_{j, \lambda}$, volume de la différence symétrique des ensembles $\tilde{\psi}_{\lambda}\left(D_{j}\right)$ et $\beta_{j, \lambda}$, majore la distance $\delta\left(\tilde{\psi}_{\lambda}\left(D_{j}\right), \beta_{j, \lambda}\right)$. Comme la distance de Hausdorff entre $\tilde{\psi}_{\lambda}\left(D_{j}\right)$ et $B_{j}$ est majorée $\operatorname{par} \delta_{\lambda}+d$ où $d$ est le diamètre euclidien d'un domaine fondamental pour le groupe de Bravais $G$, la distance $\delta\left(\tilde{\psi}_{\lambda}\left(D_{j}\right), B_{j}\right)$ tend vers 0 lorsque, à $\lambda$ fixé, $j$ tend vers l'infini.

D'après le lemme 40 , pour toute boule $\beta_{j, \lambda}$ pour la norme $|\cdot|_{1, \lambda}^{*}$, il existe une boule $\beta_{j}$ pour la norme cristalline telle que $\delta\left(\beta_{j, \lambda}, \beta_{j}\right)$ tende vers 0 lorsque $\lambda$ tend vers l'infini indépendamment de $j$. En donnant à $\lambda$ des valeurs entières et en choisissant pour chaque $\lambda$ un $j_{\lambda}$ assez grand, on obtient une sous-suite $B_{j_{\lambda}}$ qui converge vers des boules de la norme cristalline, contradiction.

\subsection{Propriétés de la norme cristalline}

On va donner une borne supérieure pour le nombre de facettes de la forme d'équilibre d'un cristal $(A, e)$ de groupe de Bravais $G$. Notons $\tilde{\Gamma}$ le graphe dont l'ensemble des sommets est $A$ et dont les arêtes correspondent aux couples $\left(a_{1}, a_{2}\right)$ tels que $e\left(a_{1}, a_{2}\right)>0$. Soit $\Gamma=\tilde{\Gamma} / G$. C'est un graphe fini qu'on peut imaginer plongé dans le tore $\mathbb{R}^{3} / G$, en réalisant ses arêtes par des segments géodésiques, éventuellement déformés pour être deux à deux disjoints. Notons $\mathcal{A}$ le nombre de sommets et $\mathcal{L}$ le nombre d'arêtes du graphe $\Gamma$.

Proposition 42. La boule unité de la norme cristalline est un polyèdre dont le nombre de facettes est au plus égal à $2^{\mathcal{L}-\mathcal{A}+2}\left(\begin{array}{c}\mathcal{L} \\ \mathcal{A}-3\end{array}\right)$, où $\mathcal{A}$ est le nombre d'atomes par cellule et $\mathcal{L}$ le nombre de liaisons par cellule. Si la fonction énergie est à valeurs rationnelles, alors les facettes sont portées par des plans rationnels.

Preuve. $\quad$ Notons $C^{0}(\Gamma, \mathbb{Z}) \simeq \mathbb{Z}^{\mathcal{A}}$ le groupe des fonctions à valeurs entières sur les sommets de $\Gamma$ et $C^{1}(\Gamma, \mathbb{Z}) \simeq \mathbb{Z}^{\mathcal{L}}$ le groupe des fonctions antisymétriques sur les arêtes orientées de $\Gamma$. La cohomologie de l'espace topologique $\Gamma$ 
peut se calculer ainsi

$$
H^{1}(\Gamma, \mathbb{Z})=C^{1}(\Gamma, \mathbb{Z}) / d C^{0}(\Gamma, \mathbb{Z})
$$

où si $g$ est une fonction sur les sommets, alors sur une arête orientée $\left(a_{1}, a_{2}\right), d g\left(a_{1}, a_{2}\right)=g\left(a_{2}\right)-g\left(a_{1}\right)$. On munit le groupe $C^{1}(\Gamma, \mathbb{Z})$ de la norme $\ell^{1}$ déterminée par la fonction énergie

$$
|c|_{1}=\sum_{\text {arêtes }} e\left(a_{1}, a_{2}\right)\left|c\left(a_{1}, a_{2}\right)\right|
$$

et le quotient $H^{1}(\Gamma, \mathbb{Z})$ de la semi-norme quotient. Le plongement de $\Gamma$ dans le tore $\mathbb{R}^{3} / G$ induit un homomorphisme

$$
h: G^{*}=H^{1}\left(\mathbb{R}^{3} / G, \mathbb{Z}\right) \rightarrow H^{1}(\Gamma, \mathbb{Z}) .
$$

La semi-norme sur $G^{*}$ duale de la norme cristalline est simplement la semi-norme induite sur $G^{*}$ par cet homomorphisme. En effet, celui-ci se relève à $C^{1}(\Gamma, \mathbb{Z})$. Soit $\ell \in G^{*}$ et $\left(a_{1}, a_{2}\right)$ une arête de $\tilde{\Gamma}$. Posons

$$
\tilde{h}(\ell)\left(a_{1}, a_{2}\right)=\ell\left(a_{2}\right)-\ell\left(a_{1}\right)
$$

Par invariance, la fonction $\tilde{h}(\ell)$ passe au quotient et devient une fonction sur les arêtes de $\Gamma$. Les "cocycles représentant $\ell$ " de la définition 4 forment le sous-espace affine $\tilde{h}(\ell)+d C^{0}(\Gamma, \mathbb{Z})$. Minimiser la norme $|\cdot|_{1}$ dans ce sous-espace affine revient à calculer la norme quotient de $h(\ell)$ dans $H^{1}(\Gamma, \mathbb{Z})$.

Celle-ci est polyédrale. En effet, la boule unité de la norme $|\cdot|_{1}$ est un polyèdre. Sa projection dans $H^{1}(\Gamma, \mathbb{R})=H^{1}(\Gamma, \mathbb{Z}) \otimes \mathbb{R}$ est un polyèdre. L'image réciproque de ce polyèdre dans $G^{*} \otimes \mathbb{R}=\left(\mathbb{R}^{3}\right)^{*}$ est un polyèdre éventuellement non borné. Par dualité, la boule unité $\beta$ de la norme cristalline est donc un polyèdre borné, éventuellement contenu dans un sous-espace vectoriel rationnel (l'image de $H_{1}(\Gamma, \mathbb{R}) \rightarrow H_{1}\left(\mathbb{R}^{3} / G, \mathbb{R}\right)$ ).

Majorons le nombre de facettes de $\beta$. Orientons arbitrairement les arêtes de $\Gamma$. La boule unité de la norme $|\cdot|_{1}$ est un octaèdre (généralisé). Ses faces de dimension $k$ sont en bijection avec les partitions de l'ensemble $\alpha$ des arêtes en trois parties $\alpha_{+}, \alpha_{-}$et $\alpha_{0}$ où $\#\left(\alpha \backslash \alpha_{0}\right)=k+1$. L'intérieur de la face associée à une telle partition est définie par les équations et inéquations

$$
c\left(a_{1}, a_{2}\right)>0(\text { resp. }<0, \text { resp. }=0) \quad \text { si } \quad\left(a_{1}, a_{2}\right) \in \alpha_{+}\left(\operatorname{resp} . \alpha_{-}, \text {resp. } \alpha_{0}\right)
$$

et

$$
\sum_{\left(a_{1}, a_{2}\right) \in \alpha_{+}} e\left(a_{1}, a_{2}\right) c\left(a_{1}, a_{2}\right)-\sum_{\left(a_{1}, a_{2}\right) \in \alpha_{-}} e\left(a_{1}, a_{2}\right) c\left(a_{1}, a_{2}\right)=1 .
$$

Le nombre de faces de dimension $k$ est $2^{k+1}\left(\begin{array}{c}\mathcal{L} \\ \mathcal{L}-1-k\end{array}\right)$.

Considérons le sous-groupe $d C^{0}(\Gamma, \mathbb{Z})+\operatorname{im}(\tilde{h}) \subset C^{1}(\Gamma, \mathbb{Z})$. Son rang vaut $\mathcal{A}+2$. Sur le sous-espace vectoriel $V$ qu'il engendre, l'octaèdre trace un polyèdre $P$. Chaque sommet de $P$ est l'intersection de $V$ avec au moins une face de dimension $k=\mathcal{L}-\mathcal{A}-2$ de l'octaèdre. La boule unité $\beta^{*}$ de la norme $|\cdot|_{\text {cris }}^{*}$ s'obtient en projetant $P$ parallèlement à $d C^{0}(\Gamma, \mathbb{R})$. Chaque sommet de $\beta^{*}$ est la projection d'au moins un sommet de $P$. Enfin chaque facette du polyèdre $\beta$ possède une équation de la forme $\ell(x)=1$ où $\ell$ est un sommet de $\beta^{*}$. Il y en a donc au plus $2^{\mathcal{L}-\mathcal{A}-1}\left(\begin{array}{c}\mathcal{L} \\ \mathcal{A}+1\end{array}\right)$.

Si $e$ est à valeurs rationnelles, alors les faces de l'octaèdre sont définies sur $\mathbf{Q}$. Comme les autres étapes de la construction ne font intervenir que des sous-espaces définis sur $\mathbf{Q}$, on conclut que les facettes de $\beta$ sont définies $\operatorname{sur} \mathbf{Q}$. 


\subsection{Exemple : le diamant}

Le diamant a pour groupe de Bravais le réseau engendré par les centres des faces d'un cube. La cellule (domaine fondamental) est la moitié de ce cube. Il y a 2 atomes et 4 liaisons par cellule. Les liaisons relient un atome à ses 4 plus proches voisins, disposés comme les sommets d'un tétraèdre régulier. Toutes les liaisons sont de force égale. Dans ce cas, $P$ cö̈ncide avec l'octaèdre régulier en dimension 4 . Sa projection $\beta^{*}$ sur $G^{*} \otimes \mathbb{R}$ est un cube et la forme d'équilibre est un octaèdre. La borne supérieure donnée par la proposition 42 est 8 . Elle est atteinte dans cet exemple.

Détaillons le calcul de la norme cristalline, en commençant par celui de la norme duale. On peut supposer que l'un des atomes est à l'origine. On note $a_{1}, a_{2}, a_{3}$ et $a_{4}$ ses plus proches voisins. Ces quatre vecteurs ont même longueur et leur somme est nulle. Soit $\ell$ une forme linéaire. Un cocycle représentant $\ell$ s'écrit $f=\ell+h$ où $h$ est une fonction $G$-invariante sur les atomes. Comme il n'y a que deux atomes par cellule, à une constante additive près, il ne reste qu'un paramètre, c'est la valeur commune $t$ des nombres $h\left(a_{i}\right)-h(0)$. La norme $|\ell|_{c r i s}^{*}$ est la borne inférieure de la fonction

$$
t \mapsto n(t)=\sum_{i=1}^{4}\left|\ell\left(a_{i}\right)+t\right| .
$$

D'après l'inégalité triangulaire, $\left|\ell\left(a_{1}\right)+t\right|+\left|\ell\left(a_{3}\right)+t\right| \geq \ell\left(a_{1}\right)-\ell\left(a_{3}\right)$ et l'égalité a lieu si $\ell\left(a_{3}\right) \leq t \leq \ell\left(a_{1}\right)$. Par conséquent, pour toute permutation $\sigma$ de $\{1,2,3,4\}$,

$$
n(t) \geq \ell\left(a_{\sigma(1)}\right)-\ell\left(a_{\sigma(3)}\right)+\ell\left(a_{\sigma(2)}\right)-\ell\left(a_{\sigma(4)}\right)=2 \ell\left(a_{\sigma(1)}+a_{\sigma(2)}\right)
$$

et l'égalité à lieu lorsque $\ell\left(a_{\sigma(4)}\right) \leq \ell\left(a_{\sigma(3)}\right) \leq t \leq \ell\left(a_{\sigma(2)}\right) \leq \ell\left(a_{\sigma(1)}\right)$. On conclut que $|\ell|_{\text {cris }}^{*}$ est le plus grand des 6 nombres de la forme $2 \ell\left(a_{\sigma(1)}+a_{\sigma(2)}\right)$. Notons $e_{i}=\frac{1}{2}\left(a_{i}+a_{4}\right)$ trois des milieux des côtés du tétraèdre formé par les plus proches voisins. Dans la base duale, la norme $|.|_{c r i s}^{*}$ est une norme $\ell^{\infty}$,

$$
|\ell|_{c r i s}^{*}=4 \max \left\{\left|\ell\left(e_{1}\right)\right|,\left|\ell\left(e_{2}\right)\right|,\left|\ell\left(e_{3}\right)\right|\right\} .
$$

Les équations des faces de sa boule unité sont de la forme $4 \ell\left(e_{i}\right)= \pm 1$. La norme duale $|\cdot|_{\text {cris }}$ est, à un facteur 4 près, la norme $\ell^{1}$ dans la base $\left(e_{1}, e_{2}, e_{3}\right)$. Les sommets de sa boule unité sont les $\pm 4 e_{i}$, i.e. les milieux des côtés du tétraèdre dilaté d'un facteur 4 .

\section{InÉgalité De SoboleV ASymptotique et homogénÉïsation}

\subsection{Version asymptotique}

Proposition 43. Soit $p>n / n-1$. La propriété isopérimétrique asymptotique

$$
\lim \inf _{a \rightarrow+\infty} I(a) / a^{n-1 / n} \geq c_{\infty}
$$

équivaut à l'inégalité de Sobolev asymptotique suivante. Pour tout $C>1 / c_{\infty}$, il existe un $C^{\prime}$ tel que, pour toute fonction u lisse à support compact,

$$
\|u\|_{L^{n / n-1}} \leq C\|d u\|_{L^{1}}+C^{\prime}\|u\|_{L^{p}} .
$$

Preave. Supposons que $\liminf I(a) / a^{n-1 / n} \geq c_{\infty}$ et soit $C>1 / c_{\infty}$. Il existe un $\alpha$ tel que $a^{n-1 / n}<C I(a)$ pour $a>\alpha$. Soit $u$ une fonction lisse à support compact. Comme $|d| u|| \leq|d u|$, on peut supposer que $u \geq 0$. Soit $\tau \in \mathbb{R}$ tel que Aire $\{u>t\} \geq \alpha$ pour $t<\tau$ et $\operatorname{Aire}\{u>\tau\} \leq \alpha$. Notons $v=\min \{u, \tau\}$. Pour $t<\tau$, notons $\chi_{t}$ la fonction caractéristique de l'ensemble de niveau $\{u>t\}$. Alors pour tout point $x$,

$$
v(x)=\int_{0}^{\tau} \chi_{t}(x) d t
$$


Il vient

$$
\|v\|_{L^{n / n-1}} \leq \int_{0}^{\tau}\left\|\chi_{t}\right\|_{L^{n / n-1}} d t=\int_{0}^{\tau}(\operatorname{Vol}\{u>t\})^{n-1 / n} d t \leq C \int_{0}^{\tau} M(\{u=t\}) d t=C\|d u\|_{L^{1}} .
$$

Comme $u-v$ est à support dans le domaine $\{u>\tau\}$ d'aire inférieure à $\alpha$, l'inégalité de Hölder donne

$$
\|u-v\|_{L^{n / n-1}} \leq \alpha^{p-n / p n}\|u-v\|_{L^{p}}
$$

d'où

avec $C^{\prime}=\alpha^{p-n / p n}$.

$$
\|u\|_{L^{n / n-1}} \leq C\|d u\|_{L^{1}}+C^{\prime}\|u\|_{L^{p}}
$$

Inversement, appliquons l'inégalité de Sobolev asymptotique à (une approximation de) la fonction caractéristique d'un domaine $D$. On trouve

$$
\operatorname{Vol}(D)^{n-1 / n} \leq C M(\partial D)+C^{\prime} \operatorname{Vol}(D)^{1 / p},
$$

d'où, si $\frac{1}{p}=\frac{1}{2}-\epsilon$,

$$
I(a) \geq \frac{1}{C} a^{n-1 / n}\left(1-C C^{\prime} a^{-\epsilon}\right) .
$$

Ceci entraîne bien que $\lim \inf _{a \rightarrow+\infty} I(a) / a^{n-1 / n} \geq c_{\infty}$.

\subsection{Homogénéisation}

Définition 44. Soit $(M, g)$ une variété riemannienne. Notons $C(M, g)$ la meilleure constante $C$ dans l'inégalité de Sobolev

$$
\|u\|_{L^{n / n-1}} \leq C\|d u\|_{L^{1}}
$$

pour les fonctions lisses à support compact sur $M$.

Notons $C_{\infty}(M, g)$ la meilleure constante $C^{\prime}$ telle que l'inégalité

$$
\|u\|_{L^{n / n-1}} \leq C^{\prime}\|d u\|_{L^{1}}
$$

soit satisfaite pour toute fonction $u$ lisse, à support compact sur $M$, et telle que $\|d u\|_{L^{\infty}} \leq 1$.

Etant donné une métrique périodique $g$ sur $\mathbb{R}^{n}$, considérons la famille des métriques dilatées $g_{t}=t^{-2} \delta_{t}^{*} g$. Comme les métriques $g_{t}$ sont homothétiques, la constante de Sobolev $C\left(\mathbb{R}^{n}, g_{t}\right)$ est indépendante de $t$. Elle vaut

$$
C\left(\mathbb{R}^{n}, g_{t}\right)=\sup \left\{a^{n-1 / n} / I(a) \mid a>0\right\}
$$

où $I$ désigne le profil isopérimétrique de $\left(\mathbb{R}^{n}, g\right)$. En revanche, la constante modifiée $C_{\infty}\left(\mathbb{R}^{n}, g_{t}\right)$ dépend de $t$. Lorsque $t$ tend vers 0 , elle tend vers $C\left(\mathbb{R}^{2}, g\right)$.

Proposition 45. $\lim \sup _{t \rightarrow+\infty} C_{\infty}\left(\mathbb{R}^{n}, g_{t}\right) \leq 1 / c_{\infty}$.

Preuve. Le profil isopérimétrique $I_{t}$ de $g_{t}$ satisfait $I_{t}(a)=t^{1-n} I\left(t^{n} a\right)$. La fonction $I_{t}(a) / a^{n-1 / n}$ converge donc vers $c_{\infty}$ lorsque $a$ tend vers $+\infty$ d'autant plus vite que $t$ est grand. Par conséquent, pour chaque $C>1 / c_{\infty}$ et $p>n / n-1$ fixés, la constante $C^{\prime}$ de l'inégalité de Sobolev asymptotique 43 pour la métrique $g_{t}$ tend vers 0 lorsque $t$ tend vers $+\infty$. Autrement dit, il existe des constantes $C_{t}$ tendant vers $c_{\infty}$ et $C_{t}^{\prime}$ tendant vers 0 telles que

$$
\|u\|_{L^{n / n-1}\left(g_{t}\right)} \leq C_{t}\|d u\|_{L^{1}\left(g_{t}\right)}+C_{t}^{\prime}\|u\|_{L^{p}\left(g_{t}\right)} .
$$


Posons $q=n p / n+p$. Comme les métriques $g_{t}$ sont uniformément équivalentes à une métrique euclidienne, l'inégalité de Sobolev

$$
\|u\|_{L^{p}\left(g_{t}\right)} \leq C^{\prime \prime}\|d u\|_{L^{q}\left(g_{t}\right)}
$$

est satisfaite avec une (mauvaise) constante indépendante de $t$. Si $u$ satisfait $\|d u\|_{L^{\infty}\left(g_{t}\right)} \leq 1$, on majore

$$
\|d u\|_{L^{q}\left(g_{t}\right)} \leq\|d u\|_{L^{1}\left(g_{t}\right)}
$$

d'où

et on obtient

$$
\|u\|_{L^{p}\left(g_{t}\right)} \leq C^{\prime \prime}\|d u\|_{L^{1}\left(g_{t}\right)}
$$

$$
\|u\|_{L^{n / n-1}\left(g_{t}\right)} \leq\left(C_{t}+C_{t}^{\prime} C^{\prime \prime}\right)\|d u\|_{L^{1}\left(g_{t}\right)}
$$

et la constante tend vers $1 / c_{\infty}$ lorsque $t$ tend vers $+\infty$.

Remarque. On peut interpréter cette proposition comme un énoncé sur l'homogénéisation de l'équation des minimisantes de la norme $L_{1}^{1}$ à norme $L^{n / n-1}$ fixée en dimension $n$ ( $c f$. [31], Chap. 5). Supposant $g=f g_{\text {eucl }}$ conforme à une métrique euclidienne (en dimension 2, ce n'est pas une restriction), on considère des fonctions $u$ uniformément lipschitziennes pour la métrique euclidienne et on examine le comportement asymptotique de la quantité

$$
\sup \frac{\sqrt{\int f(t x)^{2} u(x)^{n / n-1} d x}}{\int f(t x)|\nabla u|(x) d x}
$$

\section{BIBLIOGRAPHIE}

[1] K. Alexander, J.T. Chayes et L. Chayes, The Wulff construction and asymptotics of the finite cluster distribution for 2 dimensional Bernoulli percolation. Comm. Math. Phys. 131 (1990) 1-50.

[2] I. Babenko, Closed geodesics, asymptotic volume and characteristics of group growth. Izv. Akad. Nauk SSSR 52 (1988) 675-711; Engl. Transl. Math. USSR Izv. 33 (1989) 1-37.

[3] I. Babenko, Volume rigidity of 2-dimensional manifolds. Mat. Zametki 48 (1990) 10-14; Engl. Transl. Math. Notes 4 (1990) 629-632.

[4] W. Blaschke, Über affine Geometrie VII: Neue Extremeigenschaften von Ellipse und Ellipsoid. Ber. Verh. Sächs. Akad. Wiss. Leipzig, Math.-Phys. Kl. 69 (1917) 306-318.

[5] D.Yu. Burago et S. Ivanov, On asymptotic volume of tori. Geom. Funct. Anal. 5 (1995) 800-808.

[6] D.Yu. Burago et S. Ivanov, On asymptotic isoperimetric constant of tori (1998) preprint.

[7] H. Brunn, Über Ovale und Eiflächen. Inaug. Diss. München (1887).

[8] D.Yu. Burago, Periodic metrics. Representation theory and dynamical systems. Adv. Sov. Math. 9 (1992) 205-210.

[9] P. Curie, Sur la formation des cristaux et sur les constantes capillaires de leurs différentes faces. Bull. Soc. Minér. France 5 (1885) $145-150$.

[10] R. Cerf, Large deviation for three dimensional supercritical percolation. Prépublication d'Orsay 98.71 (1998).

[11] J. de Coninck, F. Dunlop et V. Rivasseau, On the microscopic validity of the Wulff construction and of the generalized Young equation. Comm. Math. Phys. 121 (1989) 401-419.

[12] R. Dobrushin, R. Kotecky et S. Shlosman, Wulff construction, a global shape from local interaction. Transl. Math. Monogr. 104. Providence, RI, Amer. Math. Soc. (1992).

[13] G. Faber, Beweis dass unter allen homogenen membranen von gleicher Flache und gleicher Spanne, die Kreisformige den tiefsten Grundton gibt. S. B. Math. Kl. Bayer. Akad. Wiss. (1923) 169-172.

[14] H. Federer, Geometric measure theory. Springer Verlag, Berlin, Grundlehren Band 153 (1969).

[15] H. Federer, Real flat chains, cochains and variational problems. Indiana Univ. Math. J. 24 (1974) 351-407.

[16] C.F. Gauss, Principia generalia theoriae figurae fluidorum in statu aequilibrii. C.F. Gauss Werke, Band 5, Teubner (1877) $29-77$.

[17] M. Gromov, Structures métriques pour les variétés riemanniennes, J. Lafontaine et P. Pansu, Eds., Textes Mathématiques, 1. Cedic/Fernand Nathan, Paris (1981).

[18] P.M. Gruber et J.M. Wills, Handbook of convex geometry. Volume A. North-Holland, Amsterdam (1993).

[19] E. Krahn, Über eine von Rayleigh formulierte Minimaleigenschaft der Kreise. Math. Ann. 94 (1924) 97-100.

[20] J. Lott, Remark about heat diffusion on periodic spaces. Preprint Univ. Michigan (1997).

[21] V. Mazya, Classes of domains and embedding theorems for function spaces. Dokl. Akad. Nauk USSR 133 (1960) 527-530. 
[22] J. Mather, Action minimizing invariant measures for positive definite Lagrangian systems. Math. Z. 207 (1991) 169-207.

[23] R.A. Minlos et Ya.G. Sinai, The phenomenon of phase separation in some lattice models of a gas I. Mat. Sb. 73 (1967) 375448; Math. USSR Sb. 2 (1967) 325-395; II. Tr. Mosk. Mat. Obshch. 19 (1968) 113-178; Trans. Mosc. Math. Soc. 19 (1968) $121-196$.

[24] J. Moser, On the volume element on a manifold. Trans. Amer. Math. Soc. 120 (1965) 286-294.

[25] V. Milman et G. Schechtman, Asymptotic theory of finite dimensional normed spaces. Springer, Berlin, Lecture Notes in Math. 1200 (1986).

[26] P. Pansu, Croisssance des boules et des géodésiques fermées dans les nilvariétés. Erg. Th. Dynam. Syst. 3 (1983) $415-446$.

[27] P. Pansu, Profil isopérimétrique des métriques périodiques. Prépublication d'Orsay No. $98-44$ (1998).

[28] Y. Reshetnyak, An extremal problem from the theory of convex curves. Uspekhi Mat. Nauk 8 (1953) 125-126.

[29] L.A. Santalo, Un invariante afin para los cuerpos convexos del espacio de $n$ dimensiones. Portugal. Math. 8 (1949) $155-161$.

[30] U. Schnell, Periodic sphere packings and the Wulff-shape. Beiträge Algebra Geom. 40 (1999) 125-140.

[31] J. Sanchez-Hubert et E. Sanchez-Palencia, Introduction aux méthodes asymptotiques et à l'homogénéisation. Masson, Paris (1992).

[32] J.E. Taylor, Unique structure of solutions to a class of nonelliptic variational problems. Stanford 1973. Differential Geom., Proc. Symp. Pure Math. 27 Part 1 (1975) 419-427.

[33] J.M. Wills, Lattice packings of spheres and the Wulff-shape. Mathematika 43 (1996) 229-236.

[34] G. Wulff, Zur Frage der Geschwindigkeit des Wachtums und der Auflösung der Krystalflächen. Z. Krystall. Min. 34 (1901) $449-530$. 